\title{
Pleiotropic Function of the Putative Zinc-Finger Protein MoMsn2 in Magnaporthe oryzae
}

\author{
Haifeng Zhang, ${ }^{1}$ Qian Zhao, ${ }^{1}$ Xianxian Guo, ${ }^{1}$ Min Guo, ${ }^{1}$ Zhongqiang Qi, ${ }^{1}$ Wei Tang, ${ }^{1}$ Yanhan Dong, ${ }^{1}$ \\ Wenwu Ye, ${ }^{1}$ Xiaobo Zheng, ${ }^{1}$ Ping Wang, ${ }^{2}$ and Zhengguang Zhang ${ }^{1}$ \\ ${ }^{1}$ Department of Plant Pathology, College of Plant Protection, Nanjing Agricultural University, and Key Laboratory of Integrated \\ Management of Crop Diseases and Pests, Ministry of Education, Nanjing 210095, China; ${ }^{2}$ Department of Pediatrics and \\ The Research Institute for Children, Louisiana State University Health Sciences Center, New Orleans, Louisiana 70118, U.S.A.
}

Submitted 15 September 2013. Accepted 17 December 2013.

The mitogen-activated protein kinase MoOsm1-mediated osmoregulation pathway plays crucial roles in stress responses, asexual and sexual development, and pathogenicity in Magnaporthe oryzae. Utilizing an affinity purification approach, we identified the putative transcriptional activator MoMsn2 as a protein that interacts with MoOsm1 in vivo. Disruption of the MoMSN2 gene resulted in defects in aerial hyphal growth, conidial production, and infection of host plants. Quantitative reverse transcription-polymerase chain reaction analysis showed that the expression of several genes involved in conidiophore formation was reduced in $\Delta M o m s n 2$, suggesting that MoMsn2 might function as a transcriptional regulator of these genes. Subsequently, MoCos1 was identified as one of the MoMsn2 targets through yeast one-hybrid analysis in which MoMsn2 binds to the AGGGG and CCCCT motif of the MoCOS1 promoter region. Phenotypic characterization showed that MoMsn2 was required for appressorium formation and penetration and pathogenicity. Although the $\Delta$ Momsn2 mutant was tolerant to the cell-wall stressor Calcofluor white, it was sensitive to common osmotic stressors. Further analysis suggests that MoMsn2 is involved in the regulation of the cell-wall biosynthesis pathway. Finally, transcriptome data revealed that MoMsn2 modulates numerous genes participating in conidiation, infection, cell-wall integrity, and stress response. Collectively, our results led to a model in which MoMsn2 mediates a series of downstream genes that control aerial hyphal growth, conidiogenesis, appressorium formation, cell-wall biosynthesis, and infection and that also offer potential targets for the development of new disease management strategies.

In Magnaporthe oryzae, asexual spores (conidia) are the major transmissible agent of rice blast disease. The conidia

Haifeng Zhang and Qian Zhao contributed equally to this work.

Current address for M. Guo: Department of Plant Pathology, College of Plant Protection, Anhui Agricultural University, Hefei 230036, China

Corresponding author: Z. Zhang; Email: zhgzhang@njau.edu.cn; Telephone: +186-25-84396972; Fax: +186-25-84395325.

* The $e$-Xtra logo stands for "electronic extra" and indicates that four supplementary tables and one supplementary figure are published online and that Figures 6 and 11 appear in color online.

(C) 2014 The American Phytopathological Society germinate and, then, develop a dome-shaped structure known as the appressorium, which breaches the plant cuticle through a penetration peg (Howard et al. 1991; Talbot 2003). After colonization, numerous conidia are produced on the lesions and initiate a new disease cycle. Thus, sporulation plays a key role in the infection cycle of this fungal pathogen (Zhou et al. 2009). Relatively little information exists on the regulation of conidiation in M. oryzae, and only a few genes (e.g., MoSMO, MoCON7, MoCOS1, MoCOM1, and MoHOX genes) that encode components of the conidiogenesis pathway have been identified (Liu et al. 2011a; Yang et al. 2010).

Fungi are known to sense external stresses, such as changes in $\mathrm{pH}$, dessication, and nutrient deprivation, and adapt accordingly. The budding yeast Saccharomyces cerevisiae adapts to stresses using several distinct signaling pathways (Mager and De Kruijff 1995; Nicholls et al. 2004). The yeast bZIP transcription factor (TF) Yap1 is responsible for responses to oxidative and heavy-metal stresses (Schnell et al. 1992), while two closely related, functionally redundant $\left(\mathrm{C}_{2} \mathrm{H}_{2}\right)_{2}$ zinc-finger $\mathrm{TF}, \mathrm{Msn} 2$ and Msn4, are responsible for responses to mild heat shock, starvation, osmotic stress, alcohol, and weak acids by binding to the stress response element, 5'-CCCCT-3', located in the promoters of many stress-related genes (Causton et al. 2001; Gasch et al. 2000; Martinez-Pastor et al. 1996). Stress signaling requires the $\mathrm{N}$-terminal region of $\mathrm{Msn} 2$, which includes its nuclear export sequence and a short region, designated as homology domain 1, that is highly similarity to Msn4 (Boy-Marcotte et al. 2006; Durchschlag et al. 2004; Gorner et al. 1998, 2002).

Transcriptional regulation by a diverse array of TF is a major mechanism underpinning the control of cellular development and differentiation in response to physiological or environmental stimuli. M. oryzae is thought to possess over $400 \mathrm{TF}$, but only a few of them have been characterized (Choi et al. 2009b; Guo et al. 2010; Kim et al. 2009; Odenbach et al. 2007; Park et al. 2002; Qi et al. 2012; Zhang et al. 2009; Zhou et al. 2009). The osmoregulation pathway is one of the key signaling pathways involved in the development and pathogenicity of $M$. oryzae. Deletion of MoSln1, an upstream two-component system kinase, leads to reduced growth, conidiation, and virulence, and increased sensitivity to environmental stresses (Zhang et al. 2010a). The mitogen-activated protein kinase (MAPK) MoOsm1 is essential for the response to the hyperosmotic stress but is dispensable for infection (Dixon et al. 1999). Deletion of the HIK1 (OS-1 ortholog) histidine kinase gene or the $M o S S K 1$ gene resulted in the phenotypic defect similar to that of the $\Delta$ Moosm1 mutant (Motoyama et al. 2005). However, the targets of these TF remain elusive. 
To elucidate the regulatory pathways controlling conidiation and stress response in $M$. oryzae, we identified and characterized MoMsn2, a novel zinc-finger protein that interacts with MoOsm1.

\section{RESULTS}

\section{Identification of proteins associated} with MoOsm1 in vivo.

To identify MoOsm1-interacting proteins, a MoOSM1$3 x F L A G$ construct was generated and transformed into the $M$. oryzae wild-type strain 70-15. Western blot analysis of the resulting transformant showed the presence of a 42-kDa band that is the expected size of a MoOsm1-3xFLAG fusion protein. Following affinity purification, proteins bound to the antiFLAG M2 beads were eluted and analyzed by mass spectrometry (MS) analysis. One of the MoOsm1-interacting genes was MGG_00501.7, named MoMSN2, which encodes a protein highly similar to $S$. cerevisiae Msn2, which is a well-conserved transcriptional activator with the typical $\mathrm{C}_{2} \mathrm{H}_{2}$ zinc-finger DNA-binding domains (Fig. 1A and B). In addition to MoMsn2, homologs of several ATP synthase subunits, heat-shock proteins, components of the proteasome, and a number of hypothetical proteins were also found (Supplementary Table S1).

\section{MoMsn2 physically interacts with MoOsm1.}

In $S$. cerevisiae, Hog1 (MoOsm1) is a MAPK that is involved in osmoregulation (Dixon et al. 1999) and in the interaction with Msn2 (Vendrell et al. 2011). To confirm the interaction between MoMsn2 and MoOsm1, pGADT7::MoMSN2 and pGBKT7::MoOSM1 were cotransformed into yeast cell AH109. The result showed that MoMsn2 and MoOsm1 also interacted through the yeast two-hybrid assay (Fig. 2A). In addition, the MoOSM1-3xFLAG and MoMSN2-GFP (green fluorescent protein) fusion constructs were cotransformed into protoplasts of $M$. oryzae 70-15. Transformants expressing the MoOSM13xFLAG and MoMSN2-GFP constructs were screened by the presence of the GFP signal and by Western blot analysis with an anti-FLAG antibody. In Western blot analysis with total proteins isolated from these positive transformants, the antiFLAG and anti-GFP antibodies detected the presence of 42$\mathrm{kDa}$ and $87-\mathrm{kDa}$ bands, respectively. In proteins eluted from

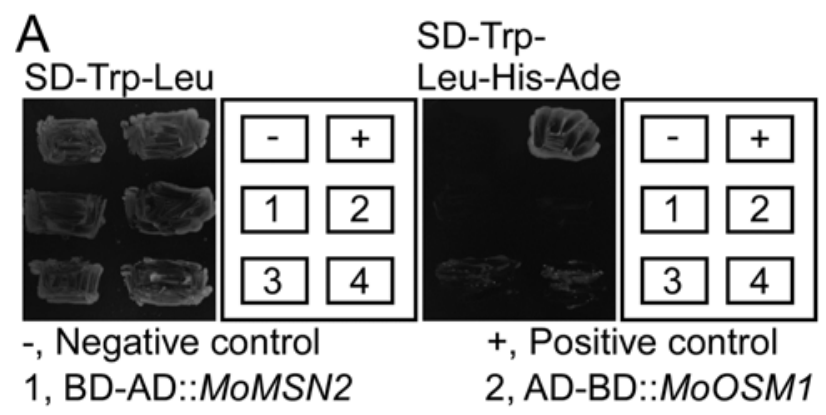

\section{3,4, AD::MoMSN2-BD::MoOSM1}

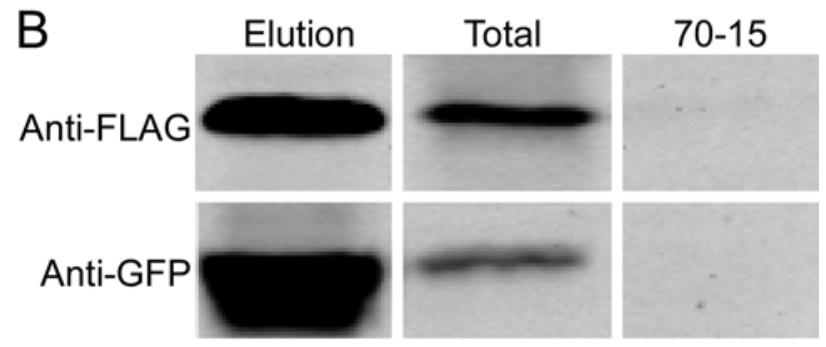

Fig. 2. MoMsn2 physically interacts with MoOsm1. A, Yeast two-hybrid assay. Yeast transformants expressing the MoMSN2 prey and MoOSM1 bait constructs were assayed for growth on SD-Trp-Leu and SD-Trp-LeuHis-Ade plates. The interaction between pGBKT7-53 and pGADT7-T was used as the positive control, and noninteractions between pGBKT7-Lam and pGADT7-T, BD (pGBKT7)-MoOsm1 and AD (pGADT7), BD and AD-MoMsn2 were used as negative controls. B, Co-immunoprecipitation assay. Western blot analysis with total proteins (Total) isolated from transformants coexpressing the MoMSN2-GFP (green fluorescent protein) and MoOSM1-3xFLAG constructs and proteins eluted from the anti-FLAG M2 beads (Elution). The presence of MoMsn2 and MoOsm1 was detected with an anti-GFP and an anti-FLAG antibody, respectively.
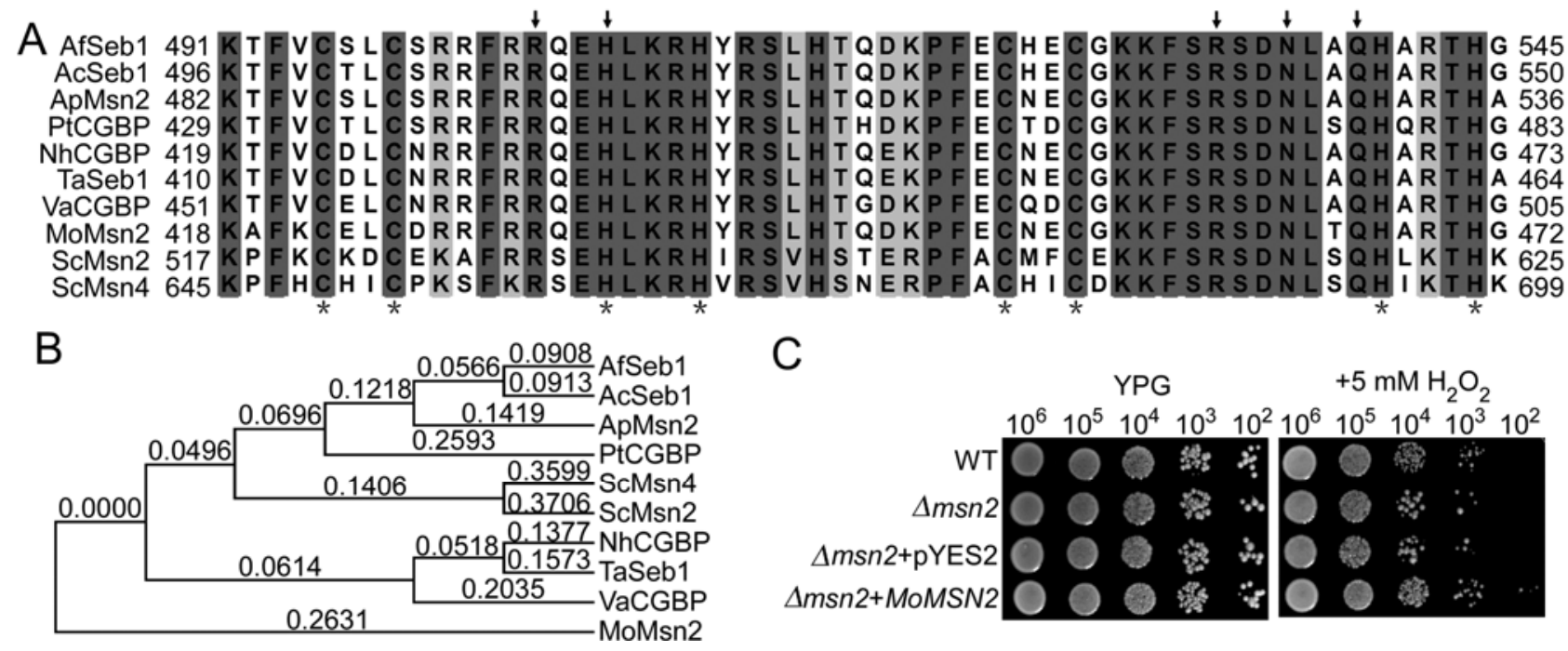

Fig. 1. Alignment and phylogenetic analysis of MoMsn2 and its orthologs and results from a yeast complementation assay. $\mathbf{A}$, Alignment of the $\left(\mathrm{C}_{2} \mathrm{H}_{2}\right)_{2}$ zinc-finger domain with similar proteins from AfSeb1 (Aspergillus fumigatus, GenBank accession number XP_751917.1), AcSeb1 (Aspergillus clavatus, XP_001271800.1), ApMsn2 (Aspergillus parasiticus, ACV03836.1), PtCGBP (Pyrenophora tritici-repentis, XP_001933138.1), NhCGBP (Nectria haematococca, AAB04132.1), TaSeb1 (Trichoderma atroviride, AAM73769.1), VaCGBP (Verticillium albo-atrum, EEY18577.1), MoMsn2 (Magnaporthe oryzae, XP_368743.1), and Saccharomyces cerevisiae (ScMsn2, NP 013751 and ScMsn4, NP 012861). The cysteine and histidine residues of the two zinc fingers are indicated by asterisks. Amino acids putatively involved in AGGGG binding are indicated by arrows. Residues conserved in all proteins are shown in gray bars. B, Phylogenetic analysis of MoMsn2 and its homologs from other organisms using a Clustal W 1.83 program. C, Yeast cells (10 ${ }^{2}$ to $10^{6}$ cells per milliliter) of wild type (WT), $\Delta m s n 2$ mutant, and $\Delta m s n 2 / M o M S N 2$ or $\Delta m s n 2 / \mathrm{pYES} 2$ transformants were assayed for growth on $1 \%$ yeast extract, $2 \%$ peptone, $3 \%$ glycerol (galactose) plates with or without $5 \mathrm{mM} \mathrm{H}_{2} \mathrm{O}_{2}$. 
the anti-FLAG M2 beads, the 87-kDa MoMsn2-GFP band was also detected with an anti-GFP antibody (Fig. 2B). These results confirmed that MoMsn2 interacts with MoOsm1.

\section{MoMSN2 rescues the defect of a $S$. cerevisiae $\Delta m s n 2$ mutant.}

The amino acid sequence of $M$. oryzae Msn2 shares $55 \%$ identity with $S$. cerevisiae Msn2. To test whether MoMsn2 is functional in $S$. cerevisiae, the MoMSN2 open reading frame was cloned into pYES2 and was transformed into a $\Delta m s n 2$ mutant of $S$. cerevisiae that is hypersensitive to oxidative stress (Fig. 1C). The resulting $\operatorname{Trp}^{+}$yeast transformants containing the pYES2-MoMSN2 construct restored growth to the $\Delta S c m s n 2$ mutant on medium containing $5 \mathrm{mM} \mathrm{H}_{2} \mathrm{O}_{2}$. In contrast, strains transformed with the empty pYES2 vector were as sensitive as the $\Delta S c m s n 2$ mutant to oxidative stress (Fig. 1C). This result indicated that MoMsn2 has the conserved function in mediating the stress-response pathway.

\section{MoMsn2 is required}

for aerial hyphal growth and conidiogenesis.

To further characterize the function of MoMsn2, the MoMSN2 gene-replacement construct was constructed and $\Delta$ Momsn 2 mutants were generated. After confirmation by Southern blot and real-time polymerase chain reaction (PCR) analysis using a gene-specific probe and primer sets (Supplementary Fig. S1; Supplementary Table S2), two $\Delta$ Momsn2 mutants were selected for phenotype characterization, along with the complemented mutant strain. The wild-type Guy11, $\Delta$ Momsn 2 mutants, and complemented transformants were inoculated onto complete medium (CM) and straw decoction and corn powder (SDC) media, respectively. After incubation at $28^{\circ} \mathrm{C}$ for 7 days, the $\Delta M o m s n 2$ mutants showed an apparent defect in radial growth, with fewer aerial hyphae and altered pigmentation (Fig. 3A and B). The biomass of the $\Delta M o m s n 2$ mutants, however, was significantly increased when cultured in liquid CM culture (Fig. 3C). Further, the mycelia mats formed by the $\Delta$ Momsn 2 mutants were more compact than that of the wild-type and complemented strains (Fig. 3D). These results indicated that MoMsn2 is required for aerial hyphal growth.

The $\Delta$ Momsn 2 mutant produced no conidia on CM and SDC plates when cultured under light for 10 days, with the exception of several elongated and thinner uniform conidia occasionally found in pre-autolysis scrapings of the SDC cultures (Fig. 4A). The $\Delta M o m s n 2$ mutant was also defective in conidial production under the induction condition of continuous illumination (Fig. 4B). When stained with lactophenol aniline blue (Song et al. 2010; Zhou et al. 2009), rare and abnormal conidiophores and conidia could be seen in the $\Delta M o m s n 2$ mutant, in contrast to the robust formation of normal conidiophores and conidia in the control strains (Fig. 4C). These results indicated that MoMsn2 is essential for conidiophore development and conidia formation in $M$. oryzae.

\section{MoMsn2 mediates the expression of several conidiogenesis-related genes.}

To reveal the mechanism by which MoMsn2 is required for conidiophore development and conidia formation, we examined the expression of several conidiation-related genes by quantitative reverse transcription (qRT)-PCR. The expression levels of MoCOS1, a $\mathrm{C}_{2} \mathrm{H}_{2}$ zinc-finger TF-encoding gene involved in the early stages of conidiation and infection (Zhou et al. 2009), and the homeobox gene MoHOX2 that is essential for conidiogenesis (Kim et al. 2009; W. Liu et al. 2010) were significantly decreased in the $\Delta M o m s n 2$ mutant (Supplementary Table S3). In contrast, the expressions of MoFLBC, MoSTUA, $M o M E D A, M o C O N 6$, and MoCON8 were remarkably increased in the $\Delta$ Momsn2 mutant (Fig. 5). These results indicated that
MoMsn2 might function as a key upstream TF in the conidiogenesis signaling pathway by modulating the expression of several conidiation-related genes.

\section{MoMsn2 targets to MoCos1 via its AGGGG motif.}

To further establish the regulation of MoCOS1 and MoHOX2 by MoMsn2, we examined the promoter regions of MoCOS1 and MoHOX2 to identify putative MoMsn2-binding sites (AG GGG or CCCCT). A sequence containing AGGGG in the $M o C O S 1$ promoter region but not that of $M o H O X 2$ was found.

To test if the AGGGG sequence is responsible for binding by MoMsn2, we conducted yeast one-hybrid and promoter mutagenesis assays. The yeast one-hybrid assay indicated that MoMsn2 directly interacts with the promoter region of MoCOS1 (Fig. 6A). We also generated two constructs in which GFP was either driven by the original MoCOS1 promoter region (pYF11::proCOS1::GFP) or the region with a mutation in the putative binding site (pYF11::proCOS1m::GFP). A strong GFP signal was observed in the transformants expressing the pYF11::proCOS1::GFP in Guy11, in contrast to the transformants expressing pYF11::proCOS1m::GFP in Guy11 or pYF11::proCOS1::GFP in the $\triangle$ Momsn2 mutant, in which no GFP signal was observed (Fig. 6B). Taken together, these results suggested that MoMsn2 functions upstream of MoCos1 by targeting the AGGGG sequence in the MoCOS1 promoter region.

\section{MoMsn2 is essential}

for appressorium formation and pathogenicity.

To investigate the function of MoMsn2 in pathogenicity, infection assays were performed on both barley and rice seedlings. Because the conidia produced by the mutants were insufficient to perform infection assays, mycelial plugs were used to inoculate 7-day-old barley leaves and 3-week-old rice leaves, respectively. The $\Delta M o m s n 2$ mutant lost the ability to cause diseases in either host, while Guy11 developed typical blight symptoms. This defect by $\Delta M o m s n 2$ was fully restored in the complemented $\Delta M o m s n 2$ mutant (Fig. 7A and B), indicating that MoMSN2 is essential for pathogenicity.

Generally, $M$. oryzae infects rice tissues through appressoria, which develop from the conidia. However, hyphae can also invade rice roots (Sesma and Osbourn 2004) and wounded leaf tissues (Zhou et al. 2009). A previous study also reported that $M$. oryzae can form hypha-driven appressoria to break the rice leaf cuticle and causes diseases (Kim et al. 2009). To investigate whether the loss of pathogenicity in the $\Delta M o m s n 2$ mutants was due to a lack of hyphae-driven appressorium formation or penetration, we evaluated the role of MoMsn2 in appressorium formation. The wild-type Guy11 freely developed fully melanized appressoria at the hyphal tips, while the $\Delta M o m s n 2$ mutant formed almost no appressoria (Fig. 7C). Therefore, we conclude that MoMsn2 is required for hyphae-driven appressorium formation, and deletion of MoMSN2 leads to inability to form appressoria, resulting in a loss of pathogenicity.

\section{MoMsn2 is crucial for invasive hyphal growth.}

The results above indicated that $\Delta$ Momsn 2 mutants had lost the ability to penetrate the plant cell. To test whether the mutants maintain their pathogenicity against abraded rice leaves, we inoculated wounded 3-week-old rice leaves with mycelial plugs. Both Guy11 and the $\Delta$ Momsn 2 mutants caused blight symptoms at the wounded sites after 5 days, but the MoMSN2 mutants induced lesions smaller than the wild-type strain (Fig. 7D), indicating that MoMSN2 is still required for invasive hyphal growth. These results suggested that MoMsn2 function is not limited to appressoria development but may include invasive growth inside the hosts. 
MoMsn2 is involved

in cell-wall integrity and stress responses.

The cell wall is central to fungal physiological processes, including $\mathrm{pH}$ and osmotic regulation and metal ion homeostasis.
Since Calcofluor white (CFW) preferentially binds to polysaccharides containing 1, 4-linked D-glucopyranosyl units and, therefore, alters the assembly of chitin microfibrils, it is often used to perturb cell-wall integrity (Egan et al. 2007). To further

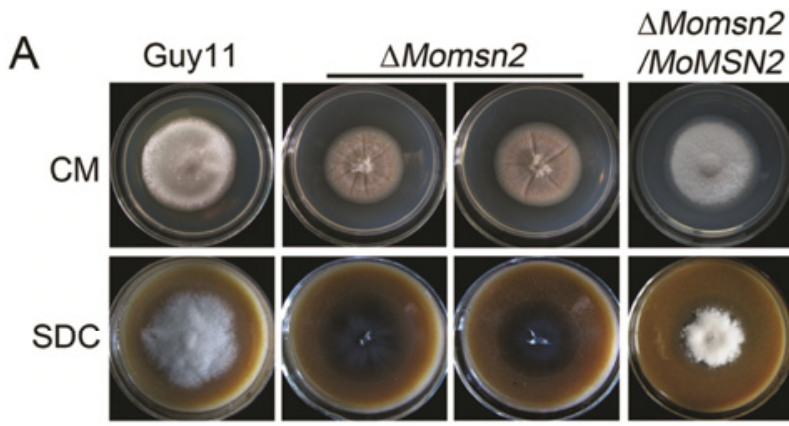

B Guy11
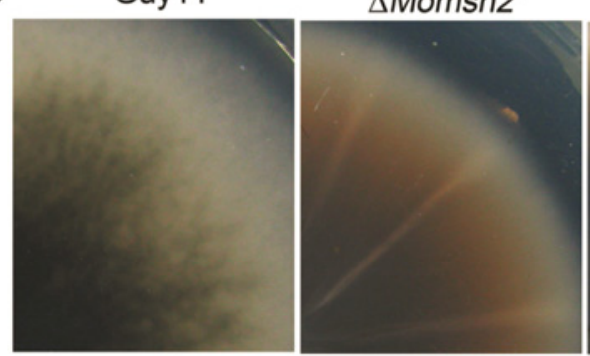

$\Delta$ Momsn2 /MOMSN2

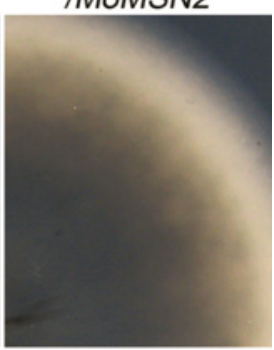

D
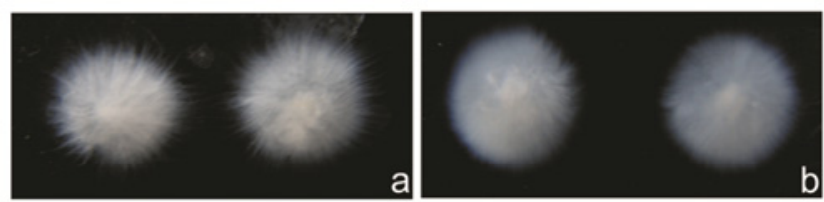

a Guy11

b $\Delta M o m s n 2$

C $\triangle$ Momsn2/MoMSN2

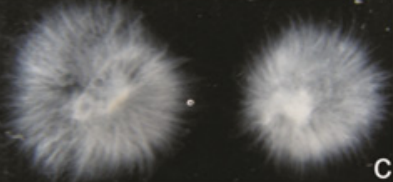

Fig. 3. MoMsn 2 is required for aerial hyphal growth. A, Colony morphology of Guy $11, \Delta M o m s n 2$, and the complemented transformant $(\Delta M o m s n 2$ / MoMSN2) grown on complete medium (CM) and straw decoction and corn powder (SDC) media plates for 7 days. B, Colony pigmentation is compromised in the $\Delta$ Momsn 2 mutants. The testing strains were cultured as described in A. C, Fungal cultures grown for various days in CM liquid media were processed for dry-weight measurement. D, Phenotype of mycelia growth in liquid CM media. The wild-type Guy11, $\Delta M o m s n 2$ mutant, and complemented transformants were inoculated in liquid CM media and were cultured for $48 \mathrm{~h}$ at $28^{\circ} \mathrm{C}$ in darkness. Three independent experiments yielded similar results.

A

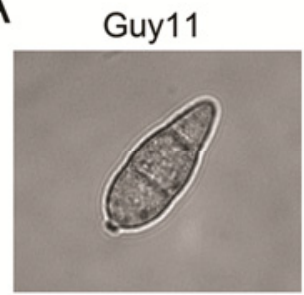

B

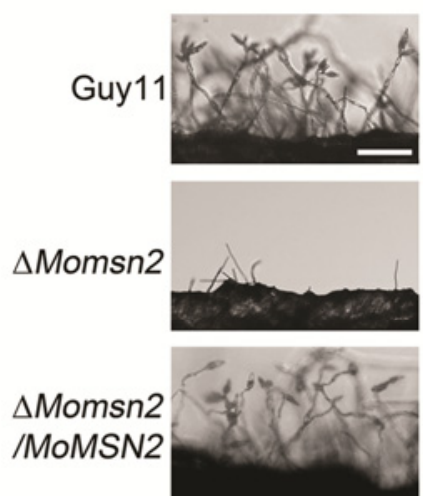

$\Delta$ Momsn2

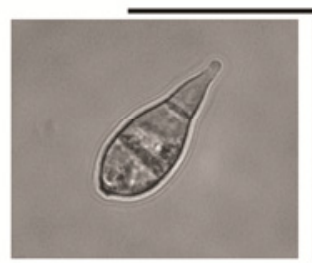

C
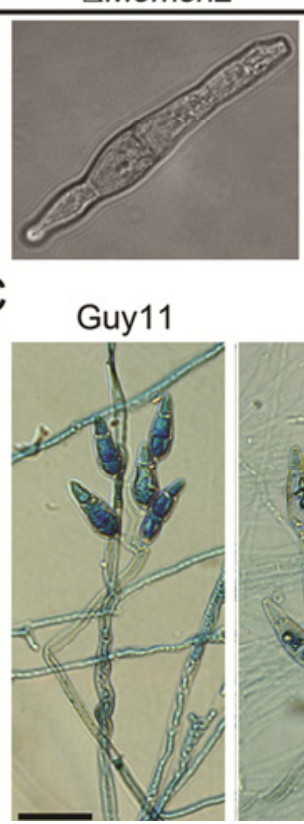

$\Delta$ Momsn2 MoMSN2

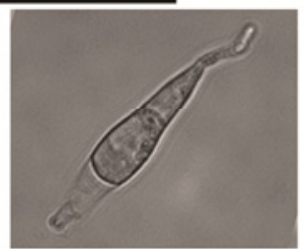

$\Delta$ Momsn2

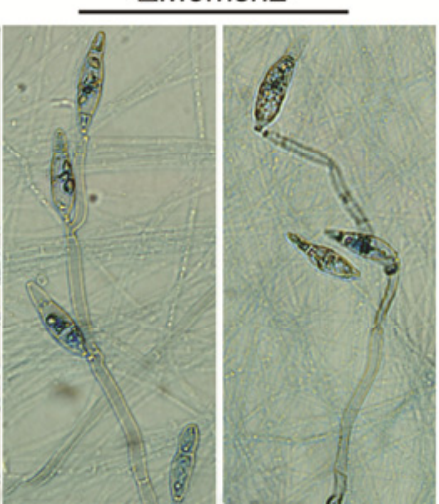

$\Delta$ Momsn2 MoMSN2

Fig. 4. MoMsn2 is required for normal conidiogenesis and conidia formation. A, Rare conidia of the $\Delta M o m s n 2$ mutant displayed abnormal morphology with elongation observed under the microscope (Ba $\mathrm{r}=5 \mu \mathrm{m})$. B, Development of conidia and conidiophores. Hyphal blocks (7-day-old) were placed on glass slides for $24 \mathrm{~h}$ to induce conidiation. (Ba $\mathrm{r}=10 \mu \mathrm{m}$ ). C, Aerial structures were stained with lactophenol aniline blue and were observed under microscope. Conidia and hyphae stained blue, whereas conidiophores stained gray. (Bars=10 $\mu \mathrm{m})$. 
understand the regulatory function of MoMsn2, we examined its role in response to CFW. Surprisingly, the $\Delta M o m s n 2 \mathrm{mu}-$ tant showed tolerance to $\mathrm{CFW}$ and was resistant to $\mathrm{CFW}$ even at the concentration of $600 \mu \mathrm{g} / \mathrm{ml}$ (Fig. 8A and B).

We then assayed the chitin content, since chitin is one of the main components of fungal cell walls (Roncero 2002). The $\Delta$ Momsn2 mutant had lower cell-wall chitin content than Guy11 (Fig. 8C). It is well-documented that the synthesis of chitin depends on the activity of the chitin synthase enzymes that are present in many fungal species, including $M$. oryzae. Therefore, we examined the expression of several genes known to be involved in chitin synthesis (MGG_01802.6, MGG_04145.6, MGG_09551.6, MGG_06064.6, MGG_ 09962.6, MGG_13013.6, and MGG_13014.6) (Kong et al.

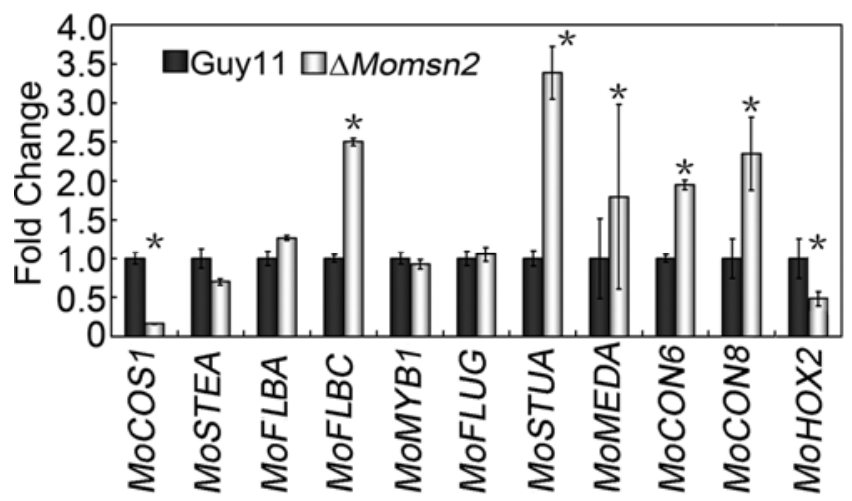

Fig. 5. Transcriptional expression of sporulation-related gene homologs in the $\Delta$ Momsn 2 mutant. Expression of genes or homologs known to be sporulation-related in Magnaporthe oryzae and other fungi. Total RNA of the mutant was extracted from mycelia grown in liquid complete medium (CM) for 3 days. Asterisks indicate a significant difference between wild type and the $\Delta$ Momsn 2 mutant $(P<0.01)$. Graphs indicate mean \pm standard error. Data comprise three independent experiments, each performed in duplicate.

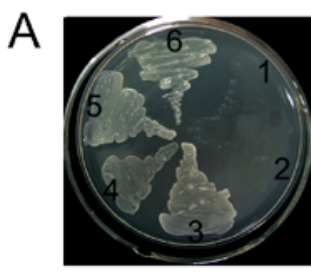

1 pAbAi::proMoCOS1

2 pAbAi::proMoCOS1+pGADT7 3 pAbAi

\section{4, 5, 6 pAbAi::proMoCOS1} +pGADT7::MOMSN2
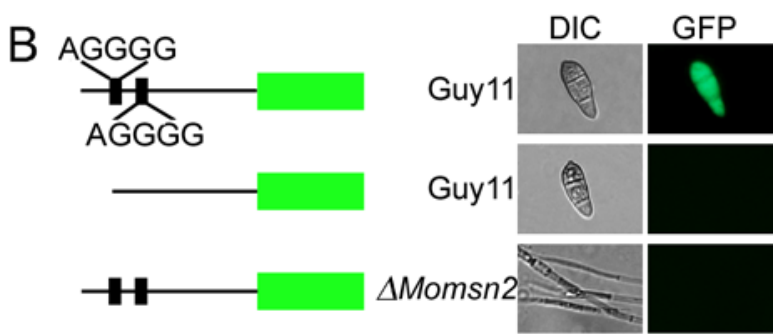

$\Delta$ Momsn2

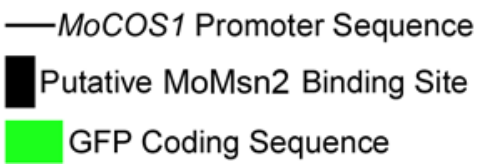

Fig. 6. MoMsn 2 binds to the promoter region of MoCOS1. A, Yeast onehybrid assay with MoCOS1 promoter as the bait and MoMsn2 as the prey. The interaction of pAbAi::proMoCOS1 and pGADT7-T was used as the negative control and pAbAi as the positive control. B, Mutation of the putative MoMsn2 binding sites in the MoCOS1 promoter region. The original MoCOS1 promoter driving the green fluorescent protein (GFP) coding sequence construct was transformed to the wild-type Guy11 and the $\Delta$ Momsn 2 mutant, respectively; the mutant MoCOS1 promoter driving the GFP construct was transformed to Guy11.
2012) in Guy11 and the $\Delta$ Momsn 2 mutant. Interestingly, the expression of these genes was significantly lower in the $\Delta M o m s n 2$ mutant than in the wild-type strain under CFW (400 $\mu \mathrm{g} / \mathrm{ml})$ treatment, whereas no significant difference in expression was found under normal (no CFW) conditions (Fig. 8D). Although the mechanism for increased tolerance to CFW in the $\Delta$ Momsn 2 mutant remains unknown, these findings are consistent with the idea that MoMsn2 responds to cell-wall stressors through regulations of chitin synthesis genes.

To investigate the role of MoMsn2 in response to salt and osmotic stresses, the wild-type Guy11, DMomsn2, and complemented transformants were exposed to conditions including ion stress $(\mathrm{NaCl})$ and osmotic stress (sorbitol). The results showed the growth rate of the $\Delta M o m s n 2$ mutant was significantly decreased in comparison with the wild-type Guy11 and the complemented transformants (Fig. 9A and B). This finding indicates that MoMsn2 has a role in the osmoregulation pathway.

\section{Deletion of MoMSN2 affected extracellular laccase and peroxidase activities.}

Previous studies showed that extracellular peroxidase activities are involved in detoxifying reactive oxygen species at the infection site and are required for infectious hyphae extension and colonization (Chi et al. 2009; Guo et al. 2010, 2011). Using Congo red as an indicator for the presence of secreted peroxidases, we initially found no significant differences in mycelial growth, but a bright halo indication of degradation developed in the wild-type strain but not the $\Delta M o m s n 2$ mutant (Fig. 10A) in later stages. To show that the $\Delta M o m s n 2$ mutant had reduced extracellular peroxidase activity, the filtrates of the $\Delta M o m s n 2$
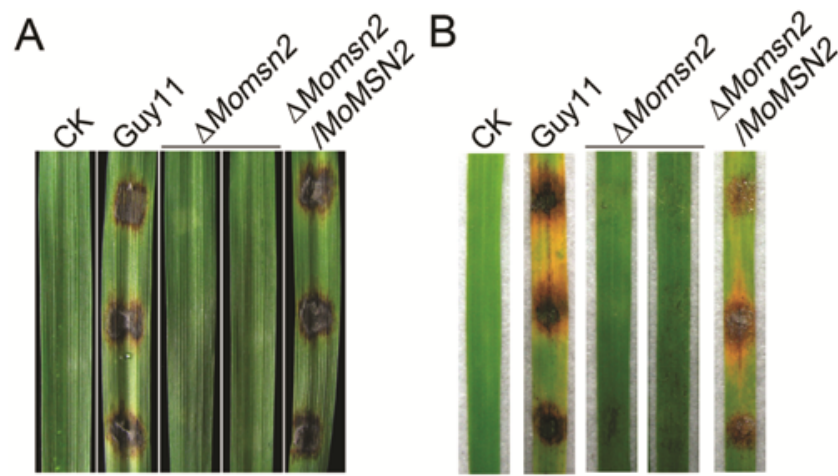

C
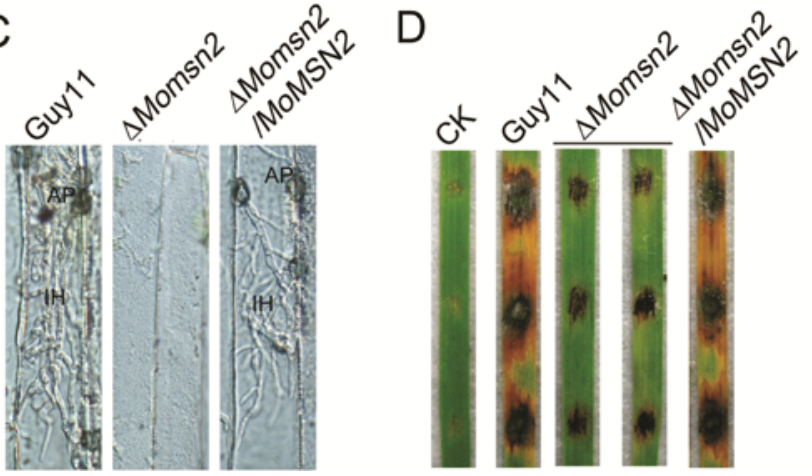

Fig. 7. Infection assays with the $\Delta$ Momsn 2 mutant. A, Intact barley and $\mathbf{B}$, rice leaves inoculated with hyphal plugs from wild-type strain Guy11, $\Delta$ Momsn2 mutant, and complemented transformants (DMomsn2/MoMSN2). C, Appressorium development on hyphal tips and infectious hyphal growth in barley leaves over $48 \mathrm{~h}$. D, Wounded rice leaves were inoculated with hyphal plugs from wild-type strain Guy11, $\Delta$ Momsn2 mutant, and complemented transformants. Inoculation with agar plugs was used as the control. Photographs were taken 3 (barley) or 5 (rice) days after inoculation. CK indicates inoculation with agar blocks. 
mutant and the Guy11 strains were collected and extracellular peroxidase activities were measured by use of a colorimetric assay. The results also showed a significant reduction of the peroxidase activity in the $\Delta$ Momsn 2 mutant (Fig. 10B). In addition, the activity of extracellular laccase was also measured on solid medium and in the culture filtrate, respectively, which showed that the $\Delta M o m s n 2$ mutant was significantly decreased in the laccase activity (Fig. 10C and D). qRT-PCR analysis revealed that the transcription of two laccase genes (MGG_11608.6 and MGG_13464.6) was significantly downregulated in the $\Delta M o m s n 2$ mutant (Fig. 10E), indicating that MoMsn2 regulates the transcription of laccase encoding genes.

\section{Expression and subcellular localization} of the MoMsn2::GFP protein in M. oryzae.

To determine expression profiles, a MoMSN2::GFP fusion construct was generated and transformed into the $\Delta M o m s n 2$ mutant. In the resulting transformants, strong GFP signals were observed in the nuclei of vegetative hyphae, mature conidia, young conidia, appressoria, and invasive hyphae (Fig. 11A). In contrast, only weak GFP signals were observed in the cytoplasm (Fig. 11A). These results indicated that MoMsn2 is constitutively expressed in the nucleus throughout the development stages. This is consistent with the important regulatory role of MoMsn2 during the development and infection stages.

In $S$. cerevisiae, the nuclear localization pattern of Msn2 was regulated by osmotic stress, heat shock, carbon-source starvation, ethanol, and sorbate (Gorner et al. 1998). To address whether MoMsn2 has a similar mechanism, conidia produced by the MoMSN2::GFP transformant were treated with $0.4 \mathrm{M} \mathrm{NaCl}$. After $30 \mathrm{~min}$, the GFP signal was much stronger and more condensed in the nucleus, and almost no GFP was observed in the cytoplasm. In contrast, the GFP signal was weak and less condensed in the nucleus in the untreated conidia under the same conditions. A weak GFP signal could also be observed in the cytoplasm (Fig. 11B). These results indicated that, consistent with ScMsn2, the nuclear localization pattern of MoMsn2 is also regulated by osmotic stress and
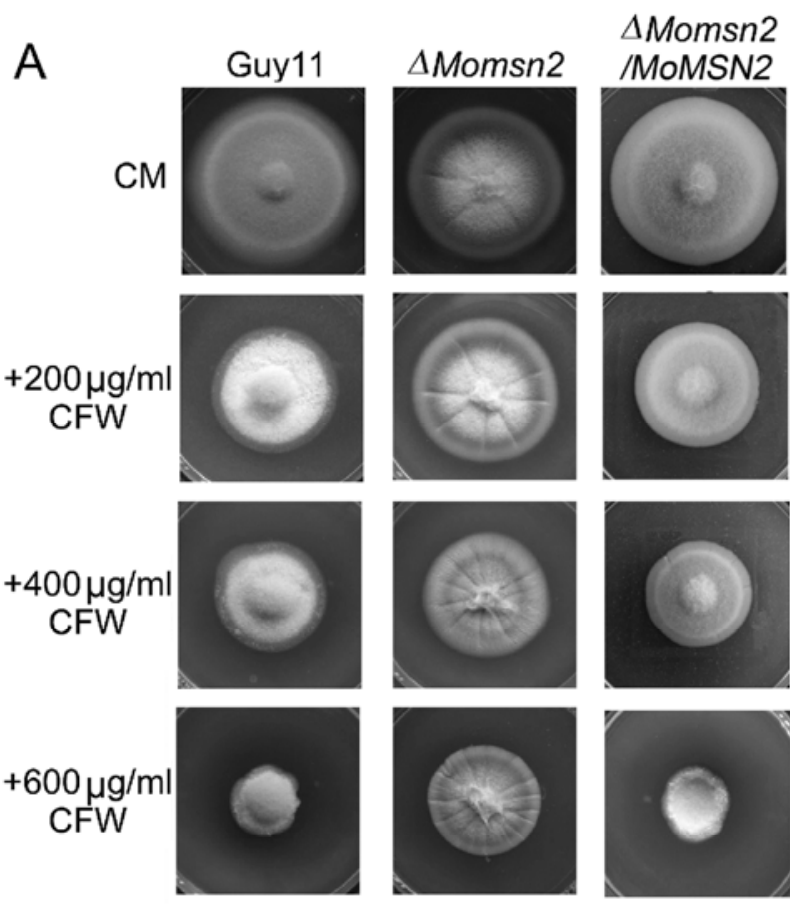

B

- Guy11 $\square \Delta$ Momsn2 $\square \Delta$ Momsn2/MoMSN2
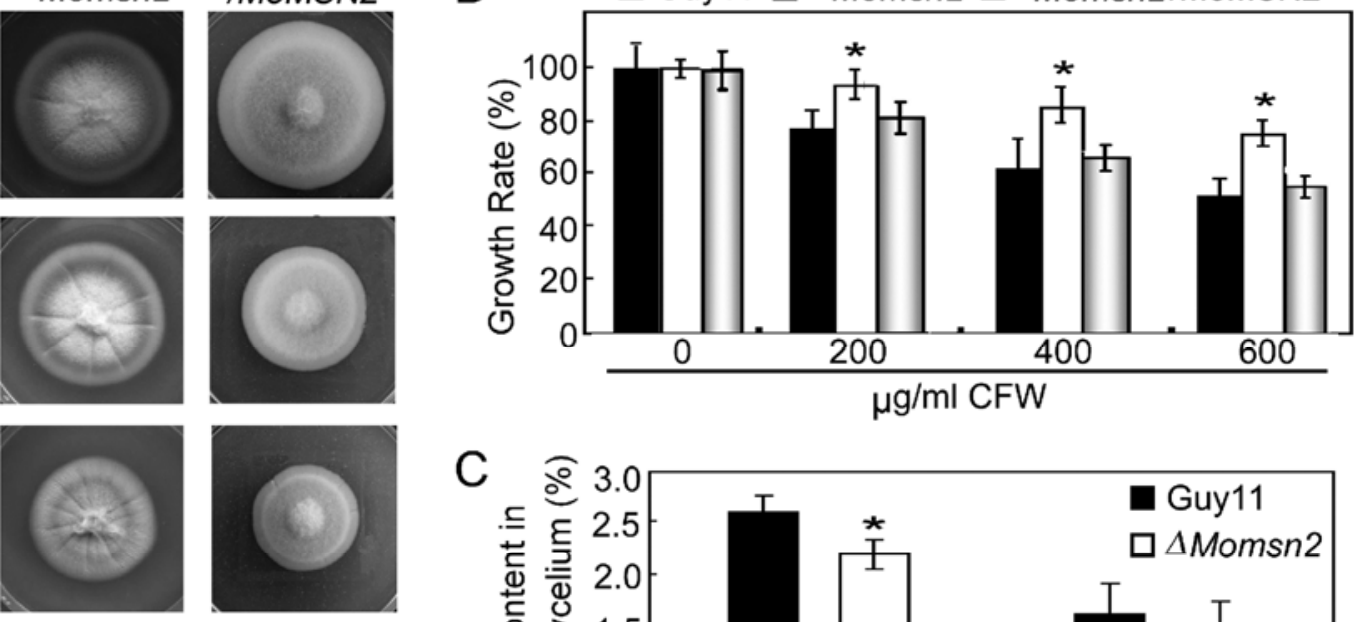

C
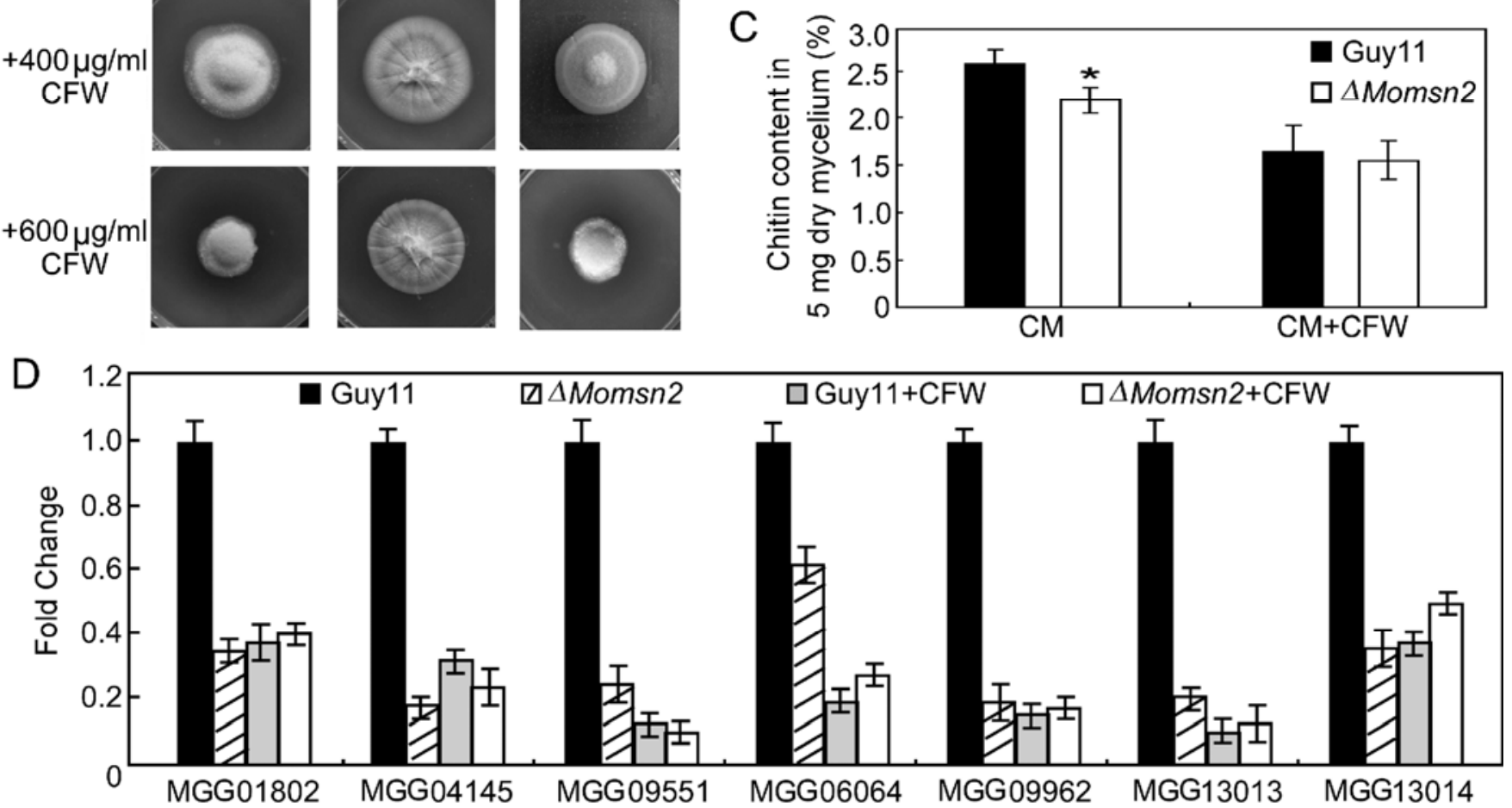

Fig. 8. MoMsn2 is involved in maintaining cell-wall integrity and controlling cellular response to Calcofluor white. A, The wild-type Guy11, $\Delta$ Momsn 2 mutant, and the complemented transformants were incubated on complete medium (CM) supplemented with different concentration of Calcofluor white (CFW) at $28^{\circ} \mathrm{C}$ for 7 days. B, Statistical analysis of the growth rate of mycelia growth on CM supplemented with CFW. C, GlcNa determination by the fluorimetric Morgan-Elson method shows reduced (no CFW treatment) or similar (CFW treatment) chitin contents in the $\Delta M o m s n 2$ mutant compared with wild type. Error bars represent the standard deviation and asterisks represent significant different among stains tested $(P=0.01)$. Data comprise three independent experiments repeated three times yielding similar results. D, The expression level of seven chitin synthases encoding genes in the $\Delta M o m s n 2$ mutant. RNA was extracted from mycelia cultured in liquid CM without or with CFW treatment for 2 days. 


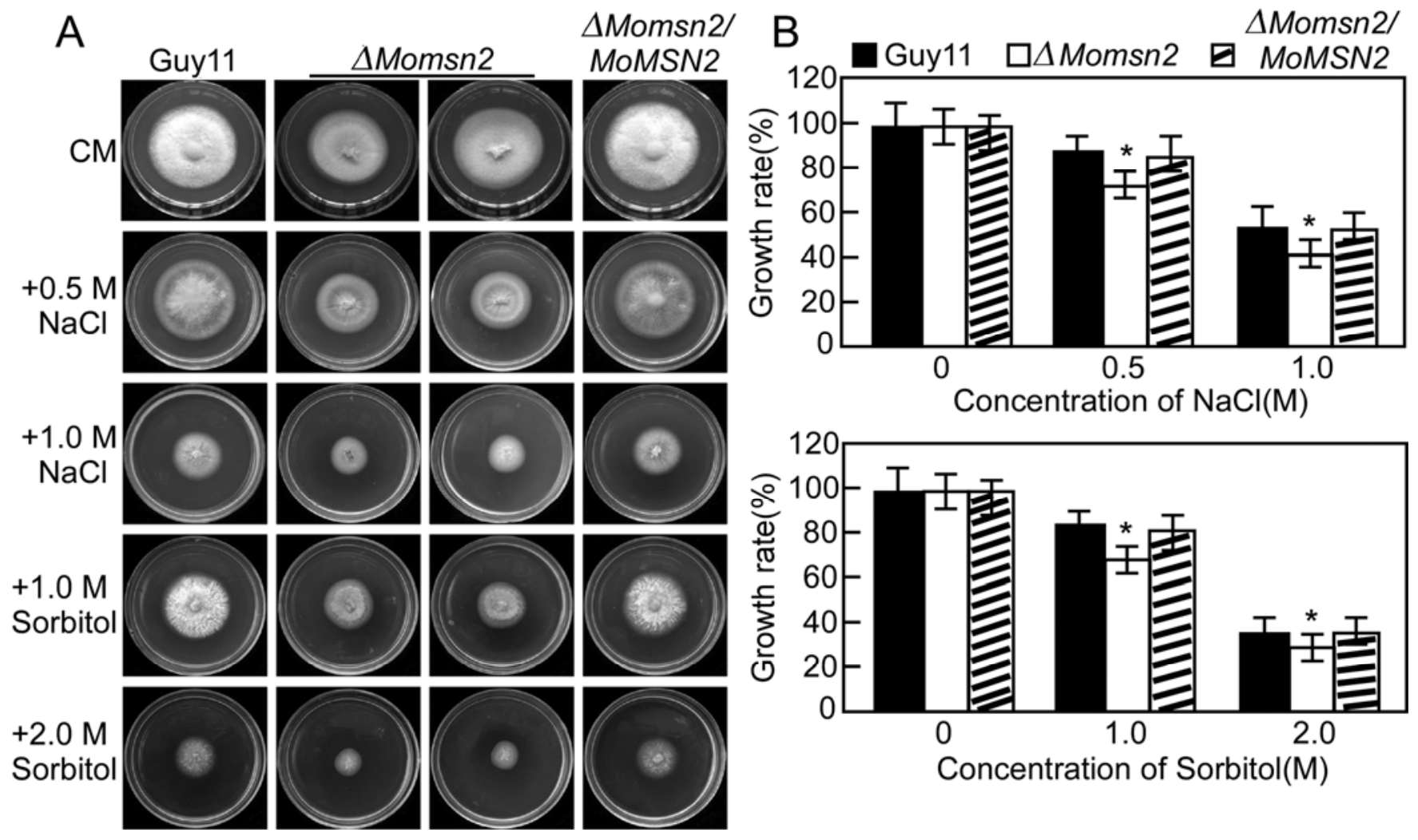

Fig. 9. Responses of wild-type, $\Delta$ Momsn2 mutant, and complemented transformant strains to high osmolarity. A, Colony morphology of Guy $11, \Delta M o m s n 2$, and the complemented transformant grown for 7 days at $28^{\circ} \mathrm{C}$ on complete medium $(\mathrm{CM})$ and $\mathrm{CM}$ containing $\mathrm{NaCl}$ or sorbitol. $\mathbf{B}$, The dose-response curve was determined 7 days after incubation at $28^{\circ} \mathrm{C}$ by plotting the percentage of colonies in the presence of various concentrations of $\mathrm{NaCl}$ or sorbitol against regular CM.

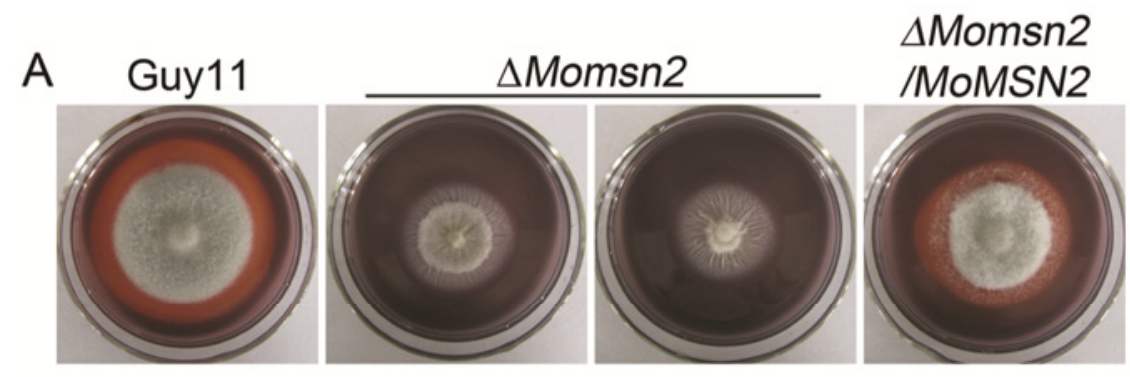

C

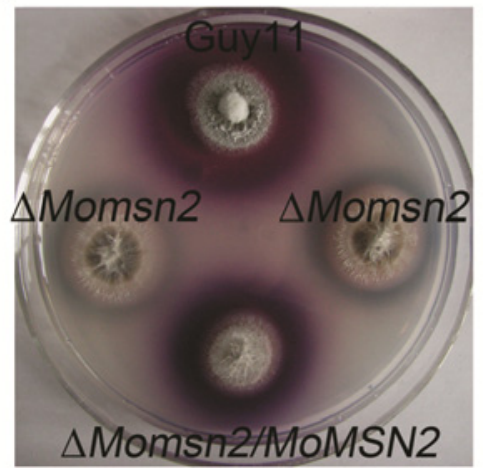

$\mathrm{D}$

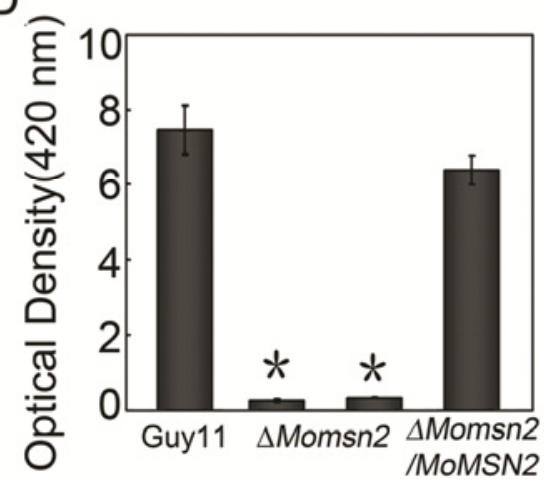

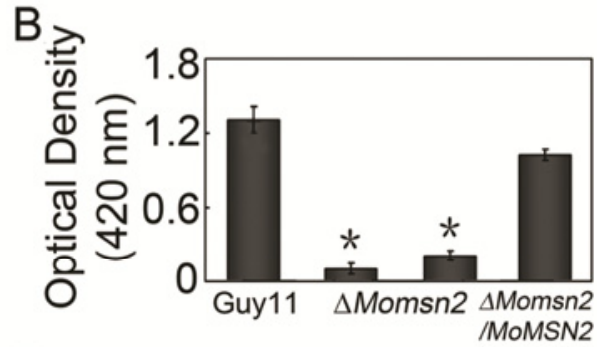

E

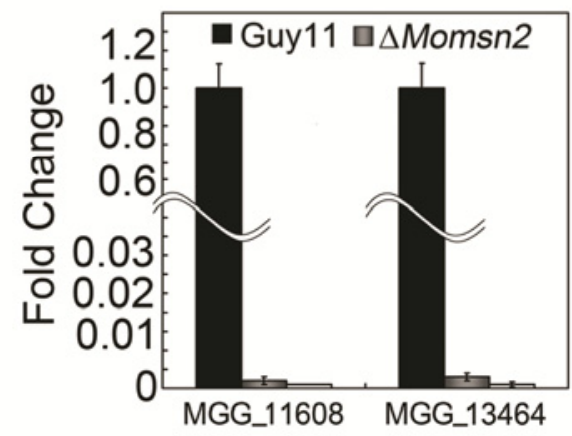

Fig. 10. Detection the activities of extracellular laccase and peroxidase in the $\Delta$ Momsn 2 mutant. A, The discoloration of Congo red was tested on complete medium (CM) plates containing dye at final concentration of $200 \mu \mathrm{g} / \mathrm{ml}$. Strains were inoculated on CM agar containing Congo red. Discoloration was observed on day 7 after inoculation and incubation at $28^{\circ} \mathrm{C}$. B, Extracellular peroxidase activity measured by an $2,2^{\prime}$-azino-di-3-ethylbenzathiazoline-6sulphonate (ABTS) oxidizing test under $\mathrm{H}_{2} \mathrm{O}_{2}$ supplemented conditions. The experiments were repeated three times. Asterisks indicate a significant difference between wild type and the $\Delta$ Momsn 2 mutant $(P=0.01)$. C, Laccase activity was tested on CM plates containing 0.2 mM ABTS and were photographed after 4 days of incubation at $28^{\circ} \mathrm{C}$. D, Laccase activity measured by an ABTS oxidizing test without $\mathrm{H}_{2} \mathrm{O}_{2}$. Error bars represent standard deviation. E, Expression levels of two laccase encoding genes in the wild type and the $\Delta$ Momsn 2 mutant. 
that MoMsn2 activates the transcription of the target genes by responding to environmental stresses.

\section{Genome-wide identification}

of genes regulated by MoMsn2.

To better understand the regulatory mechanism of MoMsn2, we analyzed the genome-wide gene-expression changes of the $\Delta$ Momsn 2 mutant in comparison with wild-type Guy11 during the hyphal stage. To confirm the gene-expression patterns, four down- and three upregulated genes were also validated by qRT-PCR. The results showed the expression pattern of each gene was consistent with that in the RNA-sequence data (Fig. 12A). Although the magnitude of fold changes was much higher than that from transcriptomic data in several cases, qRT-PCR data was consistent with that from the transcriptomic data (Fig. 12A).

To identify the genes regulated by MoMsn2, we compared the gene-expression profiles between the wild-type and $\Delta M o m s n 2$ mutant strains. Based on the $\log _{2}$ ratio ( $\Delta$ Momsn2/Guy11) values two-fold change, 1,254 genes appeared upregulated, while 322 genes were downregulated in the $\Delta$ Momsn 2 mutant (Supplementary Table S4). A survey of the whole Magnaporthe genome showed that $68.8 \%$ of the genes have putative Msn2 binding sites in their promoter region, and of these, $14.3 \%$ corresponded to differentially expressed genes in the $\Delta M o m s n 2$ mutant. The number of Msn2 binding sites in most of these genes ranged from 1 to 6 (Fig. 12B). Of the 322 downregulated genes, 226 have putative MoMsn2 binding sites, while 888 of the 1,256 upregulated genes have them (Fig. 12C). These differentially expressed genes were classified into 28 functional groups using Gene Ontology (GO) (Fig. 12D).

\section{MoMSN2 regulates a series of genes related}

to pathogenicity, cell-wall integrity, and stress response.

Because MoMsn2 plays a crucial role in pathogenicity, cellwall integrity, and stress responses, the differential expression genes (greater than twofold) were analyzed through literature with their specific functions. A total of 30 known pathogenicityassociated genes, including MoCOS1, were found in the RNA sequence data; eight of them were down-regulated and 22 were up-regulated. In addition, 19 of them have putative MoMsn2 binding sites (Table 1). Meanwhile, other pairs of genes (known genes or yeast homologs) were found to be involved in cellwall integrity and stress responses, including MoSln1, an osmosensing histidine protein kinase; MoMps1 and MoMck1, two MAPK protein kinases; and two conserved SNARE proteins, MoSec22 and MoVam7 (Table 2). Acetyltransferase MoAct, succinate-semialdehyde dehydrogenase MoSsadh, 4-aminobutyrate amino-transferase MoAat, osmosensor protein MoSho1, MADS-box transcription factor MoMcm1, catalytic subunit of calcineurin MoCna and MoSln1 were also found to be involved in stress responses (Table 3). The results above indi-
A

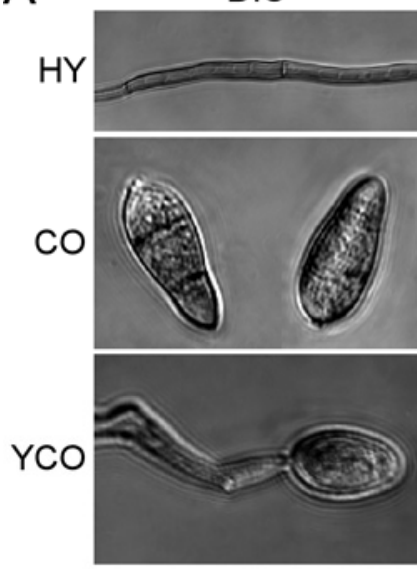

B
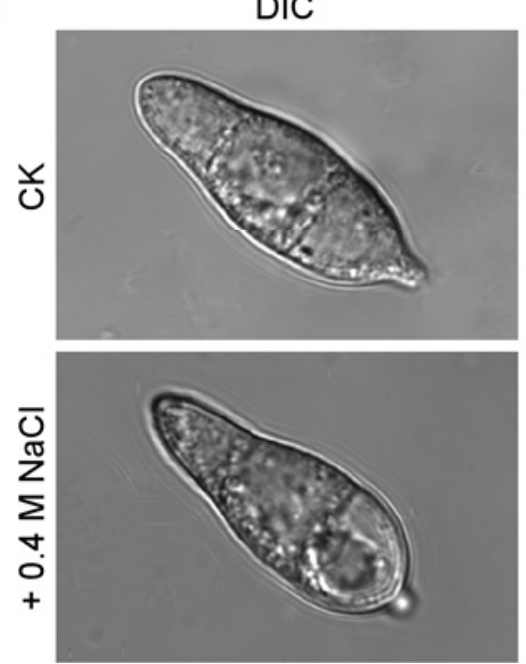

GFP

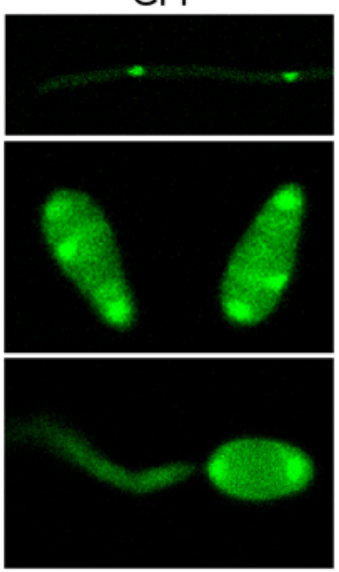

DIC
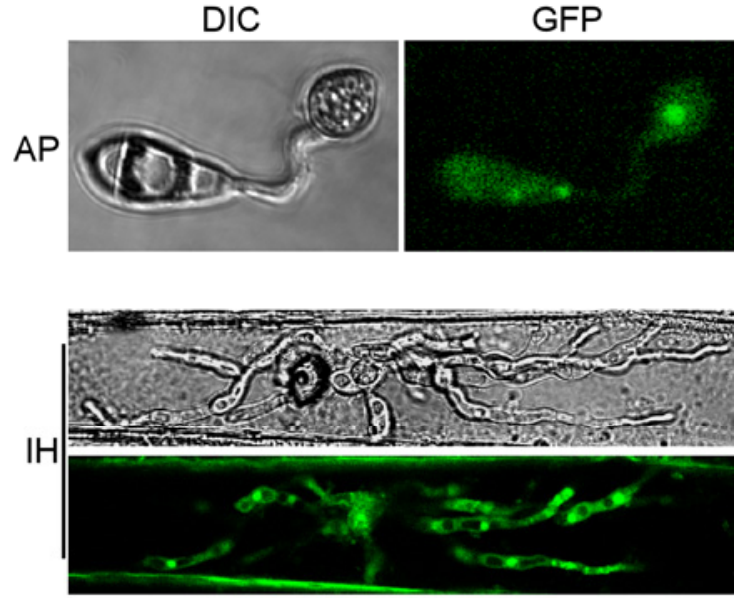

GFP
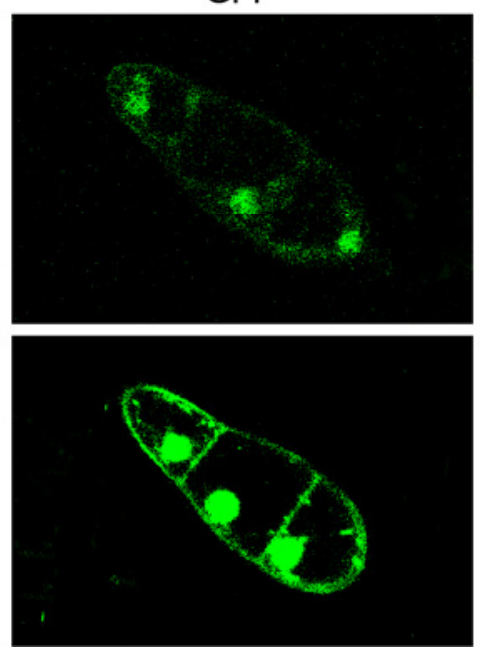

DAPI
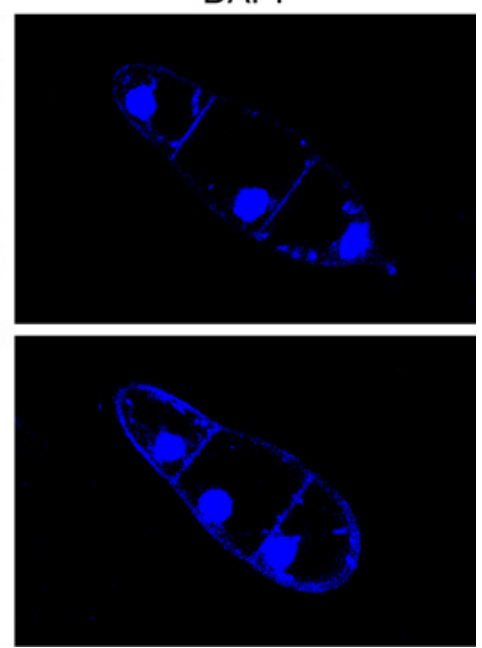

Fig. 11. Expression and localization of MoMsn2::GFP. A, Vegetative hyphae, conidia, young conidia, appressorium (12 h postinoculation [hpi]), and invasive hyphae $(24 \mathrm{hpi})$ of the transformant expressing the MoMSN2-GFP fusion construct were observed under an epifluorescence microscopy. $\mathrm{HY}=$ hyphae; $\mathrm{CO}=$ conidium; YCO = young conidium; $\mathrm{AP}=$ appressorium; $\mathrm{IH}=$ invasive hyphae. B, Epifluorescence observation of the conidia untreated (upper panels) or treated (lower panels) with $0.4 \mathrm{M} \mathrm{NaCl}$ for $30 \mathrm{~min}$. 4',6-Diamidino-2-phenylindole was added to the cultures 5 min before observation. 
cate that MoMsn2 regulates the transcription of many target genes involved in pathogenicity, cell-wall integrity, and stress response.

\section{Characterization of novel MoMsn2-regulated genes.}

To further explore the regulatory mechanisms of MoMsn2, nine putative MoMsn2 target genes (with the MoMsn2 binding site) were selected for characterization. MGG_02775 and MGG_09200 encode zinc finger $\mathrm{C}_{2} \mathrm{H}_{2}$-like proteins, $\mathrm{MGG}_{-}$ 10482 encodes a putative ketosamine-3-kinase, MGG_02805 is a magnesium ion transporter, and MGG_13815 is an $\mathrm{H}^{+}$transporting ATP synthase. In an overview of the biological phenotypes, all the target gene mutants showed no differences on vegetative growth and colony morphology compared with the wild-type strain. However, deletion of MGG_02775 leads to loss of the ability to produce conidia, which was consistent with that of the $\Delta$ Momsn2 mutant (Table 4). Meanwhile, deletion of the other target genes showed a significant reduction in conidiation (Table 4). Additionally, consistent with the
AMomsn2 mutant, the MGG_02775 and MGG_10482 deletion mutants showed more sensitivity to osmotic stressors, while the MGG_02805, MGG_13815, and MGG_09200 deletion mutants displayed no defects in response to these stressors (Table 4). These results suggested that MoMsn2 modulates various genes involved in conidium development and responses to environmental stresses.

\section{DISCUSSION}

We characterized putative $\mathrm{C}_{2} \mathrm{H}_{2}$ zinc-finger protein MoMsn2, a homolog of $S$. cerevisiae Msn2 in M. oryzae. MoMsn2 shares high sequence homologies with similar proteins found in other organisms, particularly within its zinc-finger domain (Roetzer et al. 2008). The $S$. cerevisiae Msn2 and Msn4 are transcriptional activators that respond to multiple stresses through the activation of gene transcription (Enjalbert et al. 2003; Marchler et al. 1993; Martinez-Pastor et al. 1996; Schmitt et al. 1996). In Trichoderma atroviride, the $\mathrm{C}_{2} \mathrm{H}_{2}$ zinc-finger protein $\mathrm{Seb} 1$ is
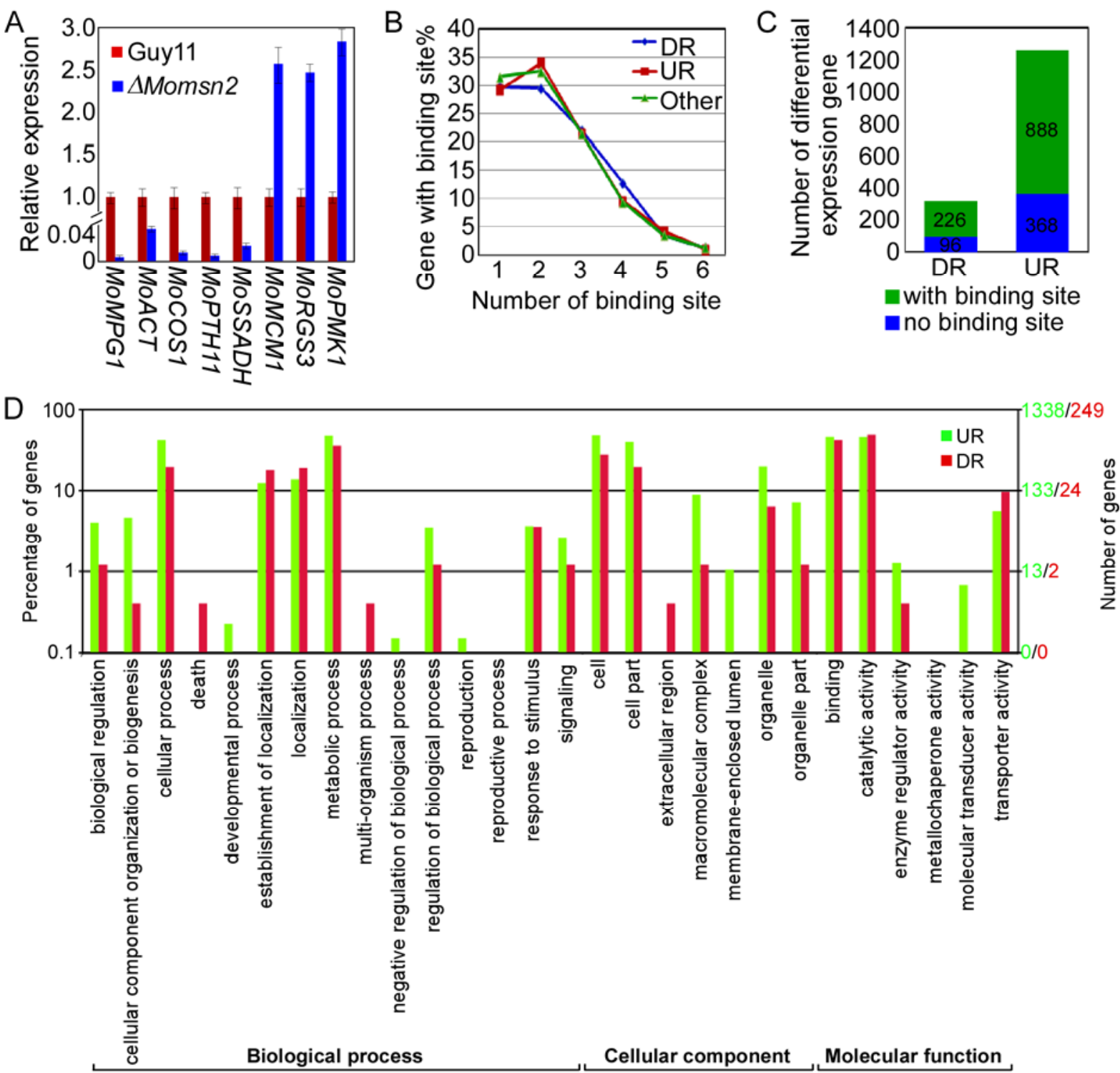

Fig. 12. Transcriptomic analyses to identify and confirm differentially expressed genes in the $\Delta M o m s n 2$ mutant and wild-type Guy11. A, Real-time reverse transcription-polymerase chain reaction to validate differential expression genes related to pathogenicity. B, The percentage of differential expression genes with binding sites. C, Number of genes with more than twofold differential expression in the $\Delta$ Momsn 2 mutant. D, Functional categorization of genes up- or downregulated in the $\Delta M o m s n 2$ mutant. The upregulated (in green) and the downregulated (in red) genes were divided into 28 groups according to their putative functions. DR = downregulation; UR = upregulation. 
involved in the response to high osmolarity (Peterbauer et al. 2002), and a G-box binding protein from Nectria haematococca, mpVI (GenBank accession number AAB 04132), is involved in the regulation of the cutinase induction (Kamper et al. 1994). Based on computer-assisted molecular modelling (Martinez-Pastor et al. 1996), we found that MoMsn2 contains conserved amino acids that bind to the target sequence AGG GG in the zinc-finger domain, which is consistent with the functions we identified through functional characterization.

Previous studies showed that fungi have evolved regulatory networks to ensure the correct timing and spatial pattern of developmental events and such developmental processes are regulated in response to stimuli from the host and the environment. Conidiogenesis and appressorium development are key steps in the colonization of host plants by many fungal pathogens, and TF play important regulatory roles in these developmental processes. We have demonstrated that MoMsn2 is a determinant of sporulation and conidiophore development by modulating the expression of several conidiation-related genes. MoMsn 2 binds to the target sequence AGGGG in the promoter region of $\mathrm{MoCos} 1$, a zinc-finger TF involved in conidiophore formation (Zhou et al. 2009), providing a functional mechanism for its regulatory function. Since no binding site was identified in the promoter region of $\mathrm{MoHOX} 2$, MoMsn 2 might likely modulate MoHox 2 indirectly or through an alternate approach. Finally, system-wide transcription analysis revealed that MoMsn2 regulates at least 30 genes involved in conidiation, appressorium formation, or virulence, singly or in combination. These data provide compelling evidence showing that MoMsn2 plays pleiotropic roles in infection-related morphogenesis and pathogenicity.

It is well-documented that wild-type strains such as Guy11 can form appressoria at the hyphal tips as a strategy for host infection (Kim et al. 2009; Song et al. 2010). Hyphal-driven appressoria cause necrotic symptoms similar to those caused by conidial-driven appressoria, even though hyphal appressorium-mediated penetration may not predominate in nature due to limitations on the spatial distribution and longevity of the hyphae. The $\Delta M o m s n 2$ mutant is defective in appressorium formation on hyphal tips, and the defect cannot be recovered by the addition of exogenous cAMP on a hydrophilic surface. This finding is consistent with the thought that MoMsn2 is

Table 1. Serial analysis of gene expression for known pathogenicity-related genes in Magnaporthe oryzae

\begin{tabular}{|c|c|c|c|c|}
\hline Gene_ID & Description & $\begin{array}{c}\log _{2} \text { ratio } \\
(\Delta M o m s n 2 / G u y 11)\end{array}$ & Reference & No. of binding sites ${ }^{a}$ \\
\hline MGG_10315 & Class I hydrophobin protein (Mpg1) & -14.0 & Talbot et al. 1993 & 1 \\
\hline MGG_05871 & Integral membrane protein (Pth11) & -6.4 & DeZwaan et al. 1999 & 2 \\
\hline MGG_15157 & Acetyltransferase (MoAct) & -5.0 & Guo et al. 2011 & 1 \\
\hline MGG_03977 & Conidiophore stalk-less 1 (Cos1) & -4.6 & Zhou et al. 2009 & 1 \\
\hline MGG_01230 & Succinate-semialdehyde dehydrogenase (MoSsadh) & -3.5 & Guo et al. 2011 & 2 \\
\hline MGG_06064 & Chitin synthase D (Chs7) & -3.3 & Kong et al. 2012 & 1 \\
\hline MGG_01662 & 4-Aminobutyrate aminotransferase(Aat) & -1.6 & Guo et al. 2011 & 1 \\
\hline MGG_09125 & Osmosensor protein (Sho1) & -1.5 & Liu et al. 2011a & 0 \\
\hline MGG_02773 & MADS-box transcription factor (MoMcm1) & 4.4 & Zhou et al. 2011b & 0 \\
\hline MGG_07312 & Osmosensing histidine protein kinase (MoSln 1$)$ & 3.4 & Zhang et al. 2010a & 0 \\
\hline MGG_09565 & CMGC/MAPK/ERK protein kinase (Pmk1) & 2.6 & Xu and Hamer 1996 & 0 \\
\hline MGG_04943 & CMGC/MAPK/ERK protein kinase (Mps1) & 2.4 & Xu et al. 1998 & 3 \\
\hline MGG_00883 & STE/STE11 protein kinase (Mck1) & 2.1 & Jeon et al. 2008 & 1 \\
\hline MGG_11346 & cAMP/PKA pathway transcriptional regulator (MoCdtf) & 2.1 & Yan et al. 2011 & 0 \\
\hline MGG_03726 & Regulator of G-protein signaling (MoRgs3) & 2.1 & Zhang et al. 2011 & 1 \\
\hline MGG_01215 & Transcription factor (Com 1$)$ & 2.0 & Yang et al. 2010 & 0 \\
\hline MGG_00527 & Extracellular Matrix Protein (Emp1) & 1.8 & Ahn et al. 2004 & 1 \\
\hline MGG_07456 & Catalytic subunit of calcineurin (MCna) & 1.7 & Choi et al. 2009a & 2 \\
\hline MGG_05428 & Conserved SNARE protein (MoVam7) & 1.6 & Dou et al. 2011 & 0 \\
\hline MGG_12958 & Transcription factor steA (Mst12) & 1.5 & Park et al. 2002 & 0 \\
\hline MGG_14008 & Regulator of G-protein signaling (MoRic8) & 1.4 & Li et al. 2010 & 3 \\
\hline MGG_00692 & Cell pattern formation-associated protein stuA (MstuA) & 1.4 & Nishimura et al. 2009 & 0 \\
\hline MGG_00990 & Regulator of G-protein signaling (MoRgs4) & 1.3 & Zhang et al. 2011 & 3 \\
\hline MGG_04050 & R-SNARE protein $(\mathrm{MoSec} 22)$ & 1.3 & Song et al. 2010 & 1 \\
\hline MGG_03046 & Sfp-type 4'-phosphopantetheinyl transferases (PPTases) (MoPpt1) & 1.3 & Horbach et al. 2009 & 1 \\
\hline MGG_04708 & cAMP/PKA pathway transcriptional regulator (MoSom1) & 1.2 & Yan et al. 2011 & 0 \\
\hline MGG_05336 & Type III transmembrane protein (Mtp1) & 1.2 & Lu et al. 2008 & 1 \\
\hline MGG_11862 & Adenylate cyclase $(\mathrm{Mac} 1)$ & 1.1 & Choi and Dean 1997 & 2 \\
\hline MGG_09912 & CAMK/CAMK1 protein kinase (MoCmk1) & 1.1 & X. Liu et al. 2010 & 1 \\
\hline MGG_10492 & Carnitine-acylcarnitine carrier protein (MoCrc1) & 1.1 & Yang et al. 2012 & 0 \\
\hline
\end{tabular}

${ }^{a}$ Number of putative MoMsn2 binding sites.

Table 2. Differentially expressed cell wall integrity-associated genes identified through serial analysis of gene expression

\begin{tabular}{|c|c|c|c|c|}
\hline Gene_ID & Description & $\log _{2} \operatorname{ratio}(\Delta$ Momsn2/Guy11) & Reference & E value \\
\hline MGG_01655 & Copper radical oxidase & -5.1 & Verna et al. 1997 & $1.10635 \mathrm{E}^{-5}$ \\
\hline MGG_07312 & Osmosensing histidine protein kinase (MoSln 1$)$ & 3.4 & Zhang et al. 2010a & 0 \\
\hline MGG_04943 & CMGC/MAPK/ERK protein kinase (Mps1) & 2.4 & Xu et al. 1998 & 0 \\
\hline MGG_00883 & STE/STE11 protein kinase (Mck1) & 2.1 & Jeon et al. 2008 & 0 \\
\hline MGG_13790 & Phosphatidylinositol 4-kinase & 1.9 & Audhya et al. 2000 & 0 \\
\hline MGG_09846 & TPR repeat-containing protein & 1.7 & Sipling et al. 2011 & 0 \\
\hline MGG_05343 & C6 zinc finger protein & 1.6 & Akache et al. 2001 & $9.51357 \mathrm{E}^{-16}$ \\
\hline MGG_05428 & Conserved SNARE protein (MoVam7) & 1.6 & Dou et al. 2011 & 0 \\
\hline MGG_04050 & R-SNARE protein $(\mathrm{MoSec} 22)$ & 1.3 & Song et al. 2010 & 0 \\
\hline MGG_01795 & AGC/PDK 1 protein kinase & 1.3 & Roelants et al. 2002 & 0 \\
\hline MGG_06599 & AGC/AKT protein kinase & 1.2 & Roelants et al. 2002 & 0 \\
\hline
\end{tabular}


involved in appressorium formation through a mechanism different from that dependent on signaling activated by cAMP.

Genome-wide analysis of $M$. oryzae cells exposed to osmotic and oxidative stress revealed that $M$. oryzae exhibits distinct functions in comparison to $S$. cerevisiae, Schizosaccharomyces pombe, and Candida albicans (Nicholls et al. 2004). In $S$. cerevisiae, there exists a general stress-mediating mechanism based on the TF Msn2 and Msn4, which are regulated by the stress (Causton et al. 2001; Estruch 2000; Gasch et al. 2000; Hohmann 2002; Martinez-Pastor et al. 1996). In C. albicans and Schizosaccharomyces pombe, Msn2-like factors do not appear to regulate the stress response. For example, $C$. albicans CaMsn4, an S. cerevisiae Msn2 ortholog, is not involved in the environmental stress response (Nicholls et al. 2004). Surprisingly, our results showed that MoMsn2 is involved in the response to multiple stresses. This might likely be due to the contribution of MoOsm1, which interacts with MoMsn2, resulting in the involvement of osmoregulation. Indeed, transcription data showed that MoMsn2 regulates many stressresponsive genes that have roles in stress responses.

In $S$. cerevisiae, the nuclear localization of Msn2 is dependent on the stress (Gorner et al. 1998). Under no-stress treatment, Msn2 is mainly distributed in the cytoplasm. In contrast, MoMsn2 was mainly found in the nuclei under normal conditions. However, both $S$. cerevisiae Msn2 and MoMsn2 showed similar nuclear localization patterns (moving from the cyto- plasm to the nuclei) under stress conditions, suggesting that, while both function as TF, MoMsn2 might possess additional functions through differentiated localization. This mechanism may result in a certain divergence in functions, including that in response to the stress. In the present study, the $\Delta M o m s n 2$ deletion mutants showed reduction in the chitin biosynthesis activity and consequent sensitive responses to several stress inducers. However, it exhibited increased tolerance to the cellwall stressor $\mathrm{CFW}$. The nature of this conflict is unclear and will be addressed in future studies.

Finally, chitin is an integral part of the fungal cell wall whose synthesis depends on the activities of multiple chitin synthase enzymes. In fungi, a number of chitin synthases that influence the outcome of the interaction with the host plant have been identified. The Botrytis cinerea class I chitin synthase Chs1 is required for virulence (Soulie et al. 2003) and the class III chitin synthase Chs3 is required for both chitin synthase activities and virulence (Soulie et al. 2006). The Fusarium oxysporum class II and V chitin synthases Chs2 and ChsV are also required for pathogenicity (Madrid et al. 2003; Martin-Udiroz et al. 2004). It is known that the class VII chitin synthase MoChs7 is required for normal chitin contents and for normal formation and function of the infection structures (Odenbach et al. 2009). In our $\Delta M o m s n 2$ mutants, the transcriptional level of seven chitin synthases was down-regulated, suggesting that MoMsn2-mediated chitin synthase activity is

Table 3. Differentially expressed stress response-associated genes identified through serial analysis of gene expression

\begin{tabular}{|c|c|c|c|c|}
\hline Gene_ID & Description & $\log _{2}$ ratio $(\Delta M o m s n 2 /$ Guy11) & Reference & E value \\
\hline MGG_15157 & Acetyltransferase (MoAct) & -5.0 & Guo et al. 2011 & 0 \\
\hline MGG_01230 & Succinate-semialdehyde dehydrogenase (MoSsadh) & -3.5 & Guo et al. 2011 & 0 \\
\hline MGG_00399 & Hypothetical protein & -2.9 & Lai et al. 2005 & $2.99975 \mathrm{E}^{-9}$ \\
\hline MGG_01819 & Glycogen phosphorylase & -2.0 & Sunnarborg et al. 2001 & 0 \\
\hline MGG_01662 & 4-Aminobutyrate aminotransferase(Aat) & -1.6 & Guo et al. 2011 & \\
\hline MGG_09125 & Osmosensor protein (Sho1) & -1.5 & Liu et al. 2011a & \\
\hline MGG_02773 & MADS-box transcription factor (MoMcm1) & 4.4 & Zhou et al. 2011b & \\
\hline MGG_07332 & Actin polymerization protein Bzz1 & 3.9 & Soulard et al. 2002 & 0 \\
\hline MGG_01719 & Electron transfer flavoprotein subunit alpha & 3.6 & Lopes et al. 2010 & 0 \\
\hline MGG_07312 & Osmosensing histidine protein kinase (MoSln 1$)$ & 3.4 & Zhang et al. $2010 \mathrm{a}$ & \\
\hline MGG_08893 & Hypothetical protein & 2.9 & Weisman and Choder 2001 & $7.067 \mathrm{E}^{-4}$ \\
\hline MGG_00501 & CGBP1 & 1.8 & Martinez-Pastor et al. 1996 & $5.92883 \mathrm{E}^{-19}$ \\
\hline MGG_07456 & Catalytic subunit of calcineurin (MCna) & 1.7 & Choi et al. 2009a & 0 \\
\hline MGG_00748 & Myosin-1 & 1.5 & Goodson et al. 1996 & 0 \\
\hline MGG_06562 & Ubiquitin-conjugating enzyme & 1.1 & Seufert and Jentsch 1990 & 0 \\
\hline MGG_09912 & CAMK/CAMK 1 protein kinase & 1.1 & Ohya et al. 1991 & 0 \\
\hline
\end{tabular}

Table 4. Characterization of the novel genes regulated by MoMsn2

\begin{tabular}{|c|c|c|c|c|c|c|c|}
\hline \multirow[b]{2}{*}{ Gene_ID } & \multirow[b]{2}{*}{ Description } & \multirow[b]{2}{*}{$\log _{2} v$} & \multirow[b]{2}{*}{ Binding Site ${ }^{w}$} & \multirow[b]{2}{*}{ Conidiation $^{x}$} & \multirow[b]{2}{*}{ Pathogenicity } & \multicolumn{2}{|c|}{ Inhibition rate on stress $(\%)$} \\
\hline & & & & & & $0.7 \mathrm{M} \mathrm{NaCl}$ & 1 M Sorbitol \\
\hline WT & Wild-type (WT) strain & - & - & $356.0 \pm 9.7$ & & $36.7 \pm 1.8 b$ & $25.0 \pm 1.2 b$ \\
\hline MGG_02775 & $\begin{array}{l}\text { Zinc finger } \mathrm{C}_{2} \mathrm{H}_{2} \text {-like protein } \\
{[\text { Myceliophthora thermophila }]}\end{array}$ & -2.4 & 2 & 0 & & $45.3 \pm 2.1 \mathrm{a}$ & $32.6 \pm 3.0 \mathrm{a}$ \\
\hline MGG_10482 & $\begin{array}{l}\text { Putative Ketosamine-3-kinase } \\
\text { [Glarea lozoyensis] }\end{array}$ & -8.7 & 1 & $232.0 \pm 14.7$ & & $45.7 \pm 1.5 \mathrm{a}$ & $33.3 \pm 1.2 \mathrm{a}$ \\
\hline MGG_02805 & $\begin{array}{c}\text { Magnesium ion transporter } \\
\text { [Grosmannia clavigera] }\end{array}$ & -5.4 & 6 & $140.7 \pm 24.4$ & & $35.8 \pm 3.8 b$ & $20.8 \pm 2.9$ \\
\hline MGG_13815 & $\begin{array}{l}\mathrm{H}^{+} \text {-transporting ATP synthase } \\
{[\text { Magnaporthe oryzae }]}\end{array}$ & -4.1 & 3 & $173.0 \pm 29.5$ & & $35.9 \pm 2.7 b$ & $22.7 \pm 2.3 b$ \\
\hline MGG_09200 & $\begin{array}{l}\text { Zinc finger protein } \\
\text { [Magnaporthe oryzae }]\end{array}$ & -3.6 & 2 & $150.2 \pm 25.5$ & $2: 00=0$ & $33.6 \pm 1.3 b$ & $26.3 \pm 2.5 b$ \\
\hline
\end{tabular}

\footnotetext{
${ }^{\mathrm{v}} \mathrm{Log}_{2}$ rate (mutant/WT).

${ }^{\text {w }}$ Putative MoMsn2 binding site.

${ }^{\mathrm{x}}$ Conidiation $\times 100 / \mathrm{cm}^{2}$.

${ }^{\mathrm{y}}$ Represents the spraying assay with conidial suspensions. All mutants showed the same pathogenicity level as wild type.

${ }^{\mathrm{z}}$ Represents the detached leaf assay with mycelia plugs, no WT image displayed here. The mutant showed the same pathogenicity level as wild type.
} 
partially responsible for the loss of pathogenicity. In addition, MoMck1 and MoMps1 MAPK kinases involved in cell-wall integrity were significantly up-regulated in the $\Delta M o m s n 2 \mathrm{mu}-$ tant through transcriptional analysis, indicating that MoMsn2 might negatively regulate MoMck1 and MoMps1 in the cellwall integrity pathway. Further studies are underway to validate such findings.

In summary, we demonstrated that MoMsn2 functions as a transcriptional regulator that plays several important functions during the development and pathogenicity of $M$. oryzae. These include regulation of asexual reproduction, appressorium development, cell-wall integrity, response to stressors, and pathogenicity (Fig. 13). This study helps unveil the regulatory mechanisms that are critical for infection-related morphogenesis and pathogenicity of the rice blast fungus.

\section{MATERIALS AND METHODS}

\section{Fungal strains, cultures, and transformation.}

$M$. oryzae Guy11 was used as the wild-type strain in this study. This and all other strains described in this study were cultured in CM (Talbot 2003). SDC medium (100 g of straw, $40 \mathrm{~g}$ of corn powder, $15 \mathrm{~g}$ of agar in 1 liter of distilled water) was used for sporulation. $M$. oryzae transformation was performed as described previously (Talbot et al. 1993). Hygromycin B (final concentration of $300 \mathrm{~g} / \mathrm{ml}$ ), sulfonylurea (final concentration of $100 \mu \mathrm{g} / \mathrm{ml}$ ), and bleomycin (final concentration of 100 $\mu \mathrm{g} / \mathrm{ml}$ ) were used as selective markers for target gene deletion, MoCOS1 overexpression, and complementation, respectively.

\section{Deletion and complementation of MoMSN2.}

To generate the $\Delta M o m s n 2$ gene deletion mutants, a 1-kb upstream flanking sequence fragment and a $1-\mathrm{kb}$ downstream flanking sequence were amplified from genomic DNA by PCR, using the primer pairs FL3959/3960 and FL3961/3962, respectively. The two flanking sequences were ligated together by overlap PCR with the primer pair FL3959/FL3962, and the approximately 2-kb amplified segments were purified and cloned into a pMD19-T simple vector (Takara Co., Dalian, China) to generate the pMD19-T::MoMSN2O vector. The hygromycin-resistance gene cassette, which encodes hygromycin phosphotransferase under the control of the Aspergillus nidulans $\operatorname{TrpC}$ promoter (Carroll et al. 1994) was amplified by PCR from plasmid pCB1003 with primer pair FL1111/FL1112 and was inserted into the EcoRV site of pMD19-T::MoMSN2O vector to generate the final deletion vector. The purified $3.4-\mathrm{kb}$ linear DNA fragment, which was amplified from the deletion vector with the primers FL3959 and FL3962, was transformed into Guy11 protoplasts. The hygromycin-resistant transformants were screened by genomic PCR and were confirmed by RT-PCR and Southern blot to obtain the gene-deletion mutants. For complementation, an approximately $3.5-\mathrm{kb}$ fragment containing the native promoter region and the entire MoMSN2 open reading frame was inserted into the pCB1532 vector, which contains a sulfonylurea-resistance cassette, and was then transformed into the $\Delta M o m s n 2$ mutant to obtain the complemented transformants.

\section{Pathogenicity assays.}

The 3-week-old seedlings of susceptible rice cultivar CO-39 and 7-day-old barley (cv. Four-arris) were used for inoculation assays as described previously (Zhang et al. 2010b). To determine the pathogenicity of the mutants, mycelia plugs of wildtype Guy11, $\Delta$ Momsn2 mutants, and complementation transformant were inoculated on the intact or wounded leaves for 3 (barley) or 5 (rice) days, were kept in a moist chamber at $28^{\circ} \mathrm{C}$ for the first $24 \mathrm{~h}$ in darkness, and were then transferred back to another moist chamber with a photoperiod of $12 \mathrm{~h}$ under lights. Lesion formation was observed daily. These experiments were all replicated three times.

\section{Measurement of laccase activity and chitin contents.}

The laccase activity on solid medium was carried out as described by Chi (2009) with little modification; a $4 \times 4 \mathrm{~mm}$ hyphal-tip plug was inoculated on CM medium supplemented with $0.2 \mathrm{mM}$ laccase substrate 2, 2'-azino-di-3-ethylbenzathiazoline6-sulfonate (Sigma Aldrich, St. Louis) and grown for 4 days. For detection of peroxidase secretion in the $\Delta$ Momsn 2 mutants, the hyphal-tip plug was placed on CM medium containing $200 \mu \mathrm{g}$ of Congo red per milliliter for 7 days. The measurement of peroxidase and laccase activity in culture filtrate was performed as described by Chi and associates (2009). Chitin ( $N$-acetylglucosamine, GlcNAc) content was determined also as described previously (Dou et al. 2011; Song et al. 2010; Zhang et al. 2010b). The experiment was repeated three times.

\section{Nucleic acid manipulation, Southern blot and real-time qRT-PCR.}

DNA extraction was performed as described by Talbot and associates (1993). DNA and RNA were manipulated using standard procedures. For qRT-PCR, $5 \mu \mathrm{g}$ of total RNA were reverse transcribed into first-strand cDNA using the oligo(dT) primer with the synthesis system of M-MLV reverse transcriptase (Invitrogen, Carlsbad, CA, U.S.A.) according to manufacturer's instructions. qRT-PCR was performed following previously established procedures (Guo et al. 2010), and the relative abundance of target transcripts was normalized to that of the actin gene (MGG_03982), also as previously described (Guo et al. 2010). Genomic DNA for PCR screening was isolated by the quick-extraction procedure as described (Joao Ramalhosa et al. 2009).

\section{Yeast one- and two-hybrid assays.}

The Matchmaker Gold yeast one-hybrid system (Clontech, Mountain View, CA, U.S.A.) was used to determine DNA-protein interactions. The MoMSN2 cDNA, amplified with primer pair FL5242/FL5243, was cloned into the pGADT7 vector (Clontech) as the prey, while the MoCOS1 promoter, amplified

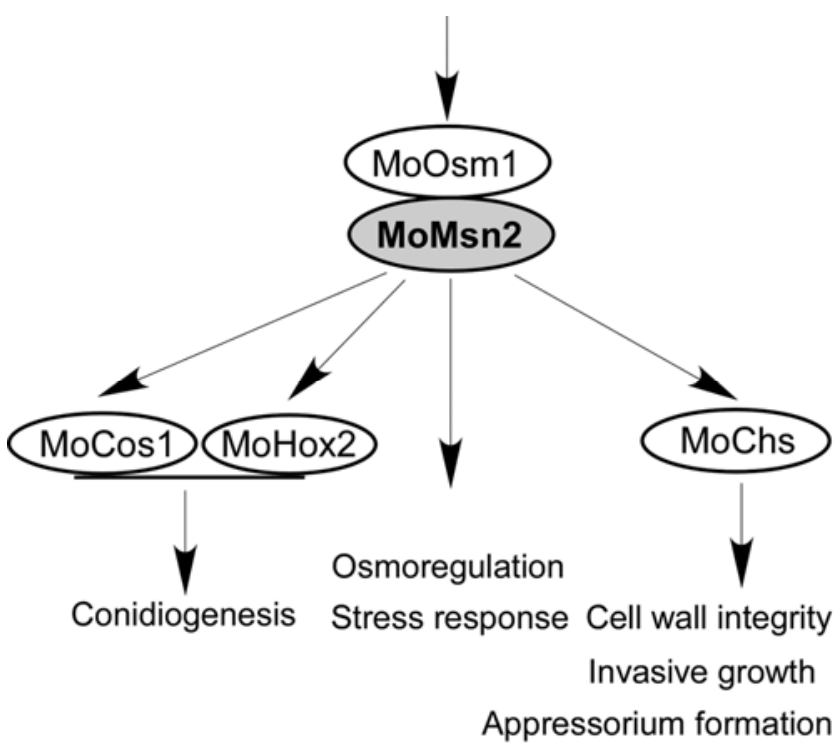

Fig. 13. A hypothetical model for MoMsn2 function in Magnaporthe oryzae. MoMsn2 interacts with MoOsm1 and regulates an array of genes including $\mathrm{MoCOS1}, \mathrm{MoHOX} 2$, and $\mathrm{MoCHS}$ to control conidiogenesis, stress response, cell-wall integrity, appressorium formation, and invasive growth. 
with primer pair FL5173/FL5174, was cloned into the pAbAi vector (Clontech) as the bait in the yeast one-hybrid system. The confirmed bait vector was first transformed into the yeast strain Y1HGold (Clontech) and the transformants harboring the bait vector were isolated with $\mathrm{Ura}^{-}$synthetic defined (SD) media and were validated by genomic PCR. Then, the purified prey vector was transformed into the validated transformants harboring the bait vector to obtain the Aureobasidin A (AbA)resistant transformants. Positive clones were further confirmed by plating onto Ura ${ }^{-} \mathrm{SD}$ media containing $\mathrm{AbA}$ to check $\mathrm{AbA}^{\mathrm{r}}$ reporter gene expression.

The bait construct was generated by cloning MoOSM1 fulllength cDNA into pGBKT7. The MoMSN2 cDNA was cloned into pGADT7 as the prey constructs. The resulting prey and bait constructs were confirmed by sequencing analysis and were transformed in pairs into yeast strain AH109 as the described by the BD library construction and screening kit (Clontech). The $\mathrm{Trp}^{+}$and $\mathrm{Leu}^{+}$transformants were isolated and assayed for growth on SD-Trp-Leu-His-Ade medium. Yeast strains for positive and negative controls were from the twohybrid assay kit.

\section{Generation of MoMSN2::GFP, MoMSN2::3xFLAG, and $M o C O S 1^{\triangle A G G G G}:: G F P$ fusion constructs.}

The entire MoMSN2 gene with its native promoter region was amplified and cloned into the pYF11 and pHZ126 vectors by the yeast gap-repair approach (Park et al. 2004). A similar approach was used to generate the $M o C O S 1^{\triangle \mathrm{AGGGG}}:: \mathrm{GFP}$ construct, which deleted the putative MoMsn2-binding site in the promoter region of MoCOS1. The resulting pYF11:: MoMSN2::GFP and pHZ126::MoMSN2::3xFLAG fusion constructs were confirmed by sequencing analysis and were transformed into the $\Delta M o m s n 2$ mutant and strain $70-15$, respectively. The resulting transformants were examined for GFP signals under an epifluorescence microscope or the expression of MoMSN2::3xFLAG fusion proteins was detected with a monoclonal anti-FLAG antibody (Sigma Aldrich).

\section{Coimmunoprecipitation and affinity purification assay.}

To confirm the interaction between MoMSN2 and MoOSM1 in vivo, the entire MoOSM1 gene was cloned into pHZ126 by the yeast gap-repair approach (Park et al. 2004). The resulting construct pHZ126::MoOSM1::3xFLAG was cotransformed with pYF11::MoMSN2::GFP into protoplasts of strain 70-15. Total proteins were isolated from transformants expressing both MoOSM1::3xFLAG and MoMSN2::GFP and were incubated with anti-FLAG M2 affinity resins (Sigma Aldrich). Proteins bound to M2 resins were eluted after a series of washing steps as described previously (Ding et al. 2010; Zhou et al. 2011a). Western blots of total proteins and elution from the M2 resins were detected with anti-FLAG (Sigma Aldrich) and anti-GFP (Sigma Aldrich) antibodies. For affinity purification, total proteins were isolated from transformants expressing the MoOSM1-3xFLAG construct and were incubated with antiFLAG M2 affinity resins. After washing three times with $1 \times$ Tris-buffered saline ( $\mathrm{pH} 7.4$ ), three times with $50 \mathrm{mM}$ trimethylammonium bicarbonate buffer, and three times with doubledistilled $\mathrm{H}_{2} \mathrm{O}$, proteins were eluted with $0.1 \%$ Rapigest as described previously (Liu et al. 2011b). The elution proteins were analyzed by Nanoflow liquid chromatography tandem MS. The resulting MS/MS data were used to search against the nonredundant $M$. oryzae protein database at the National Center for Biotechnology Information (NCBI).

\section{Bioinformatics.}

The full sequence of MoMsn2 was downloaded from the $M$. oryzae database. The Msn2 protein sequences from different organisms were obtained from the GenBank database, using the BLAST algorithm (McGinnis and Madden 2004). Sequence alignments were performed using the Clustal_W 1.83 program (Thompson et al. 1994), and the phylogenetic tree was generated by the Mega4.0 Beta program (Tamura et al. 2007). Domain architecture was predicted by the EBI online database. For GO and functional annotation, orthologs were identified between $M$. oryzae predicted proteins and proteins in the GO database. Results from local alignment using BLAST, functional domain comparisons from NCBI, and a manual literature review were used to make final assignments to $\mathrm{GO}$ functional categories.

\section{ACKNOWLEDGMENTS}

This research was supported by the National Science Foundation for Distinguished Young Scholars of China (grant number 31325022 to Z. Zhang), National Basic Research Program of China (grant number 2012CB114000, Z. Zhang), Natural Science Foundation of China (grant number 31271998, Z. Zhang), the Fundamental Research Funds for the Central Universities (grant number KYZ201304 to H. Zhang). Research in the Wang laboratory was supported by the Research Institute for Children, Children's Hospital of New Orleans, LA, U.S.A.

\section{LITERATURE CITED}

Ahn, N., Kim, S., Choi, W., Im, K. H., and Lee, Y. H. 2004. Extracellular matrix protein gene, EMP1, is required for appressorium formation and pathogenicity of the rice blast fungus, Magnaporthe grisea. Mol. Cells 17:166-173.

Akache, B., Wu, K., and Turcotte, B. 2001. Phenotypic analysis of genes encoding yeast zinc cluster proteins. Nucleic Acids Res. 29:2181-2190.

Audhya, A., Foti, M., and Emr, S. D. 2000. Distinct roles for the yeast phosphatidylinositol 4-kinases, Stt4p and Pik1p, in secretion, cell growth, and organelle membrane dynamics. Mol. Biol. Cell 11:26732689.

Boy-Marcotte, E., Garmendia, C., Garreau, H., Lallet, S., Mallet, L., and Jacquet, M. 2006 The transcriptional activation region of Msn2p, in Saccharomyces cerevisiae, is regulated by stress but is insensitive to the cAMP signalling pathway. Mol. Genet. Genomics 275:277-287.

Carroll, A. N., Sweigard, J. A., and Valent, B. 1994. Improved vectors for selecting resistance to hygromycin. Fungal Genet. Newsl. 41:22.

Causton, H. C., Ren, B., Koh, S. S., Harbison, C. T., Kanin, E., Jennings, E. G., Li, T .I., True H. L., Lander, E. S., and Young, R. A. 2001. Remodeling of yeast genome expression in response to environmental changes. Mol. Biol. Cell 12:323-337.

Chi, M. H., Park, S. Y., Kim, S., and Lee, Y. H. 2009. A novel pathogenicity gene is required in the rice blast fungus to suppress the basal defenses of the host. PLoS Pathog. 5:e1000401. Published online.

Choi, J., Kim, Y., and Lee, Y. H. 2009a. Functional analysis of MCNA, a gene encoding a catalytic subunit of calcineurin, in the rice blast fungus Magnaporthe oryzae. J. Microbiol. Biotechnol. 19:11-16.

Choi, J., Kim, Y., Kim, S., Park, J., and Lee, Y. H. 2009b. MoCRZ1, a gene encoding a calcineurin-responsive transcription factor, regulates fungal growth and pathogenicity of Magnaporthe oryzae. Fungal Genet. Biol. 46:243-254.

Choi, W., and Dean, R. A. 1997. The adenylate cyclase gene MACl of Magnaporthe grisea controls appressorium formation and other aspects of growth and development. Plant Cell 9:1973-1983.

DeZwaan, T. M., Carroll, A. M., Valent, B., and Sweigard, J. A. 1999. Magnaporthe grisea pth11 is a novel plasma membrane protein that mediates appressorium differentiation in response to inductive substrate cues. Plant Cell 11:2013-2030.

Ding, S. L., Liu, W., Iliuk, A., Ribot, C., Vallet, J., Tao, A., Wang, Y., Lebrun, M. H., and Xu, J. R. 2010. The Tig1 histone deacetylase complex regulates infectious growth in the rice blast fungus Magnaporthe oryzae. Plant Cell 22:2495-2508.

Dixon, K. P., Xu, J. R., Smirnoff, N., and Talbot, N. J. 1999. Independent signaling pathways regulate cellular turgor during hyperosmotic stress and appressorium-mediated plant infection by Magnaporthe grisea. Plant Cell 11:2045-2058.

Dou, X. Y., Wang, Q., Qi, Z. Q., Song, W. W., Wang, W., Guo, M., Zhang, H. F., Zhang, Z. G., Wang, P., and Zheng, X. B. 2011. MoVam7, a conserved SNARE involved in vacuole assembly, is required for growth, endocytosis, ROS accumulation, and pathogenesis of Magnaporthe oryzae. PLoS One 6:e16439. Published online. 
Durchschlag, E., Reiter, W., Ammerer, G., and Schuller, C. 2004. Nuclear localization destabilizes the stress-regulated transcription factor Msn2. J. Biol. Chem. 279:55425-55432.

Egan, M. J., Wang, Z. Y., Jones, M. A., Smirnoff, N., and Talbot, N. J. 2007. Generation of reactive oxygen species by fungal NADPH oxidases is required for rice blast disease. Proc. Natl. Acad. Sci. U.S.A 104:11772-11777.

Enjalbert, B., Nantel, A., and Whiteway, M. 2003. Stress-induced gene expression in Candida albicans: Absence of a general stress response. Mol. Biol. Cell 14:1460-1467.

Estruch, F. 2000. Stress-controlled transcription factors, stress-induced genes and stress tolerance in budding yeast. FEMS (Fed. Eur. Microbiol. Soc.) Microbiol. Rev. 24:469-486.

Gasch, A. P., Spellman, P. T., Kao, C. M., Carmel-Harel, O., Eisen, M. B. Storz, G., Botstein, D., and Brown, P. O. 2000. Genomic expression programs in the response of yeast cells to environmental changes. Mol. Biol. Cell 11:4241-4257.

Goodson, H. V., Anderson, B. L., Warrick, H. M., Pon, L. A., and Spudich, J. A. 1996. Synthetic lethality screen identifies a novel yeast myosin I gene (MYO5): Myosin I proteins are required for polarization of the actin cytoskeleton. J. Cell Biol. 133:1277-1291.

Gorner, W., Durchschlag, E., Martinez-Pastor, M. T., Estruch, F., Ammerer, G., Hamilton, B., Ruis, H., and Schuller, C. 1998. Nuclear localization of the $\mathrm{C}_{2} \mathrm{H}_{2}$ zinc finger protein Msn2p is regulated by stress and protein kinase A activity. Genes Dev. 12:586-597.

Gorner, W., Durchschlag, E., Wolf, J., Brown, E. L., Ammerer, G., Ruis, H., and Schuller, C. 2002. Acute glucose starvation activates the nuclear localization signal of a stress-specific yeast transcription factor. EMBO (Eur. Mol. Biol. Organ.) J. 21:135-144.

Guo, M., Guo, W., Chen, Y., Dong, S. Zhang, X., Zhang, H., Song, W. Wang., W., Wang., Q., Lv, R., Zhang, Z., Wang, Y., and Zheng, X. 2010. The basic leucine zipper transcription factor Moatf1 mediates oxidative stress responses and is necessary for full virulence of the rice blast fungus Magnaporthe oryzae. Mol. Plant-Microbe Interact. 23:1053-1068.

Guo, M., Chen, Y., Du, Y., Dong, Y., Guo, W., Zhai, S., Zhang, H. F. Dong, S. M., Zhang, Z. G., Wang, Y. C., Wang, P., and Zheng, X. B 2011. The bZIP transcription factor MoAP1 mediates the oxidative stress response and is critical for pathogenicity of the rice blast fungus Magnaporthe oryzae. PLoS Pathog. 7:e1001302. Published online.

Hohmann, S. 2002. Osmotic stress signaling and osmoadaptation in yeasts. Microbiol. Mol. Biol. Rev. 66:300-372.

Horbach, R., Graf, A., Weihmann, F., Antelo, L., Mathea, S., Liermann, J. C., Opatz, T., Thines, E., Aquirre, J., and Deising, H. B. 2009. Sfp-Type 4 '-phosphopantetheinyl transferase is indispensable for fungal pathogenicity. Plant Cell 21:3379-3396.

Howard, R. J., Ferrari, M. A., Roach, D. H., and Money, N. P. 1991. Penetration of hard substrates by a fungus employing enormous turgor pressures. Proc. Natl. Acad. Sci. U.S.A. 88:11281-11284

Jeon, J., Goh, J., Yoo, S., Chi, M.-H., Choi, J., Rho, H.-S., Park, J., Han, S.-S., Kim, B. R., Park, S.-Y., Kim, S., and Lee, Y.-H. 2008. A putative MAP kinase kinase kinase, $M C K 1$, is required for cell wall integrity and pathogenicity of the rice blast fungus, Magnaporthe oryzae. Mol. Plant-Microbe Interact. 21:525-534.

Joao Ramalhosa, M., Paiga, P., Morais, S., Delerue-Matos, C., and Prior Pinto Oliveira, M. B. 2009. Analysis of polycyclic aromatic hydrocarbons in fish: Evaluation of a quick, easy, cheap, effective, rugged, and safe extraction method. J. Sep. Sci. 32:3529-3538.

Kamper, J. T., Kamper, U., Rogers, L. M., and Kolattukudy, P. E. 1994 Identification of regulatory elements in the cutinase promoter from Fusarium solani f. sp. pisi (Nectria haematococca). J. Biol. Chem. 269:9195-9204

Kim, S., Park, S. Y., Kim, K. S., Rho, H. S., Chi, M. H., Choi, J., Park, J., Kong, S., Park, J., Goh, J., and Lee, Y. H. 2009. Homeobox transcription factors are required for conidiation and appressorium development in the rice blast fungus Magnaporthe oryzae. PLoS Genet. 5:e1000757. Published online.

Kong, L., Yang, J., Li, G., Qi, L., Zhang, Y., Wang, C., Zhao, W., Xu, J., and Peng, Y. 2012. Different chitin synthase genes are required for various developmental and plant infection processes in the rice blast fungus Magnaporthe oryzae. PLoS Pathog. 8:e1002526. Published online.

Lai, L. C., Kosorukoff, A. L., Burke, P. V., and Kwast, K. E. 2005. Dynamical remodeling of the transcriptome during short-term anaerobiosis in Saccharomyces cerevisiae: Differential response and role of Msn2 and/or Msn4 and other factors in galactose and glucose media. Mol. Cell Biol. 25:4075-4091.

Li, Y., Yan, X., Wang, H., Liang, S., Ma, W.-B., Fang, M.-Y., Talbot, N. J., Wang, Z.-Y. 2010. MoRic8 is a novel component of G-protein signaling during plant infection by the rice blast fungus Magnaporthe oryzae. Mol. Plant-Microbe Interact. 23:317-331.
Liu, W., Xie, S., Zhao, X., Chen, X., Zheng, W., Lu, G., Xu, J.-R., and Wang, Z. 2010. A homeobox gene is essential for conidiogenesis of the rice blast fungus Magnaporthe oryzae. Mol. Plant-Microbe Interact. 23:366-375.

Liu, X., Lu, J., Dong, B., Gu, Y., and Lin, F. 2010. Disruption of $M o C M K 1$, encoding a putative calcium/calmodulin-dependent kinase, in Magnaporthe oryzae. Microbiol. Res. 165:402-410.

Liu, W., Zhou, X., Li, G., Li, L., Kong, L., Wang, C., Zhang, H., and Xu, J. 2011a. Multiple plant surface signals are sensed by different mechanisms in the rice blast fungus for appressorium formation. PLoS Pathog. 7:e1001261. Published online.

Liu, W. D., Iliuk, A., Tao, A., and Ding, S. L. 2011b. Identifying protein complexes by affinity purification and mass spectrometry analysis in the rice blast fungus. Pages 157-166 in: Fungal Genomics. J. R. Xu and H. B. Bluhm, eds. Humana Press, New York.

Lopes, J., Pinto, M. J., Rodrigues, A., Vasconcelos, F., and Oliveira, R. 2010. The Saccharomyces cerevisiae genes, AIM45, YGR207c/CIR1 and YOR356w/CIR2, are involved in cellular redox state under stress conditions. Open Microbiol. J. 4:75-82.

Lu, Q., Lu, J., Li, X., Liu, X., Min, H., and Lin, F. 2008. Magnaporthe oryzae MTP1 gene encodes a type III transmembrane protein involved in conidiation and conidial germination. J. Zhejiang Univ. Sci. B 9:511519.

Madrid, M. P., Di Pietro, A., and Roncero, M. I. 2003. Class V chitin synthase determines pathogenesis in the vascular wilt fungus Fusarium oxysporum and mediates resistance to plant defence compounds. Mol Microbiol. 47:257-266.

Mager, W. H., and De Kruijff, A. J. 1995. Stress-induced transcriptional activation. Microbiol. Rev. 59:506-531.

Marchler, G., Schuller, C., Adam, G., and Ruis, H. 1993. A Saccharomyces cerevisiae UAS element controlled by protein kinase A activates transcription in response to a variety of stress conditions. EMBO (Eur. Mol. Biol. Organ.) J. 12:1997-2003.

Martin-Udiroz, M., Madrid, M. P., and Roncero, M. I. 2004. Role of chitin synthase genes in Fusarium oxysporum. Microbiol. 150:3175-3187.

Martinez-Pastor, M. T., Marchler, G., Schuller, C., Marchler-Bauer, A., Ruis, H., and Estruch, F. 1996. The Saccharomyces cerevisiae zinc finger proteins Msn2p and Msn4p are required for transcriptional induction through the stress response element (STRE). EMBO (Eur. Mol. Biol. Organ.) J. 15:2227-2235.

McGinnis, S., and Madden, T. L. 2004. BLAST: At the core of a powerful and diverse set of sequence analysis tools. Nucleic Acids Res. 32:W2025 .

Motoyama, T., Kadokura, K., Ohira, T., Ichiishi, A., Fujimura, M., Yamaguchi, I., and Kudo, T. 2005. A two-component histidine kinase of the rice blast fungus is involved in osmotic stress response and fungicide action. Fungal Genetic Biol. 42:200-212.

Nicholls, S., Straffon, M., Enjalbert, B., Nantel, A., Macaskill, S., Whiteway, M., and Brown, A. J. 2004. Msn2- and Msn4-like transcription factors play no obvious roles in the stress responses of the fungal pathogen Candida albicans. Eukaryot. Cell 3:1111-1123.

Nishimura, M., Fukada, J., Moriwaki, A., Fujikawa, T., Ohashi, M., Hibi, T., and Hayashi, N. 2009. Mstu1, an APSES transcription factor, is required for appressorium-mediated infection in Magnaporthe grisea. Biosci. Biotechnol. Biochem. 73:1779-1786.

Odenbach, D., Thines, E., Anke, H., and Foster, A. J. 2009. The Magnaporthe grisea class VII chitin synthase is required for normal appressorial development and function. Mol. Plant Pathol. 10:81-94.

Odenbach, D., Breth, B., Thines, E., Weber, R. W., Anke, H., and Foster, A. J. 2007. The transcription factor Con7p is a central regulator of infection-related morphogenesis in the rice blast fungus Magnaporthe grisea. Mol. Microbiol. 64:293-307.

Ohya, Y., Kawasaki, H., Suzuki, K., Londesborough, J., and Anraku, Y. 1991. Two yeast genes encoding calmodulin-dependent protein kinases. Isolation, sequencing and bacterial expressions of $C M K 1$ and $C M K 2$. J. Biol. Chem. 266:12784-12794.

Park, G., Xue, C., Zheng, L., Lam, S., and Xu, J.-R. 2002. MST12 regulates infectious growth but not appressorium formation in the rice blast fungus Magnaporthe grisea. Mol. Plant-Microbe Interact. 15:183-192.

Park, G., Bruno, K. S., Staiger, C. J., Talbot, N. J., and Xu, J.-R. 2004 Independent genetic mechanisms mediate turgor generation and penetration peg formation during plant infection in the rice blast fungus. Mol. Microbiol. 53:1695-1707.

Peterbauer, C. K., Litscher, D., and Kubicek, C. P. 2002. The Trichoderma atroviride seb1 (stress response element binding) gene encodes an AG GGG-binding protein which is involved in the response to high osmolarity stress. Mol. Genet. Genomics 268:223-231.

Qi, Z. Q., Wang, Q., Dou, X. Y., Wang, W., Zhao, Q., Lv, R. L., Zhang, H. F., Zheng, X. B., Wang, P., Zhang, Z. G. 2012. MoSwi6, an MAP kinase Mps1 interacting APSES family transcription factor, is required 
for hyphal and conidial morphogenesis, appressorial function, and pathogenicity of Magnaporthe oryzae. Mol. Plant Pathol 13:677-689.

Roelants, F. M., Torrance, P. D., Bezman, N., and Thorner, J. 2002. Pkh1 and $\mathrm{Pkh} 2$ differentially phosphorylate and activate $\mathrm{Ypk} 1$ and $\mathrm{Ykr} 2$ and define protein kinase modules required for maintenance of cell wall integrity. Mol. Biol. Cell 13:3005-3028.

Roetzer, A., Gregori, C., Jennings, A. M., Quintin, J., Ferrandon, D., Butler, G., Kuchler, K., Ammerer, G., and Schuller, C. 2008. Candida glabrata environmental stress response involves Saccharomyces cerevisiae Msn2/4 orthologous transcription factors. Mol. Microbiol. 69:603-620.

Roncero, C. 2002. The genetic complexity of chitin synthesis in fungi. Curr. Genet. 41:367-378.

Schmitt, A. P., and McEntee, K. 1996. Msn2p, a zinc finger DNA-binding protein, is the transcriptional activator of the multistress response in Saccharomyces cerevisiae. Proc. Natl. Acad. Sci. U.S.A. 93:57775782.

Schnell, N., Krems, B., and Entian, K. D. 1992. The PAR1 (YAP1/SNQ3) gene of Saccharomyces cerevisiae, a c-jun homologue, is involved in oxygen metabolism. Curr. Genet. 21:269-273.

Sesma, A., and Osbourn, A. E. 2004. The rice leaf blast pathogen undergoes developmental processes typical of root-infecting fungi. Nature 431:582-586.

Seufert, W., and Jentsch, S. 1990. Ubiquitin-conjugating enzymes UBC4 and UBC5 mediate selective degradation of short-lived and abnormal proteins. EMBO (Eur. Mol. Biol. Organ.) J. 9:543-550.

Sipling, T., Zhai, C., and Panaretou, B. 2011. Emw1p/YNL313cp is essential for maintenance of the cell wall in Saccharomyces cerevisiae. Microbiol. 157:1032-1041.

Song, W. W., Dou, X. Y., Qi, Z. Q., Wang, Q., Zhang, X., Zhang, H. F., Guo, M., Dong, S. M., Zhang, Z. G., Wang, P., and Zheng, X. B. 2010. R-SNARE homolog MoSec22 is required for conidiogenesis, cell wall integrity, and pathogenesis of Magnaporthe oryzae. PLoS One 5:e13193. Published online.

Soulard, A., Lechler, T., Spiridonov, V., Shevchenko, A., Li, R., and Winsor, B. 2002. Saccharomyces cerevisiae Bzz1p is implicated with type I myosins in actin patch polarization and is able to recruit actinpolymerizing machinery in vitro. Mol. Cell Biol. 22:7889-7906.

Soulie, M. C., Piffeteau, A., Choquer, M., Boccara, M., and Vidal-Cros, A. 2003. Disruption of Botrytis cinerea class I chitin synthase gene Bcchs I results in cell wall weakening and reduced virulence. Fungal Genet. Biol. 40:38-46.

Soulie, M. C., Perino, C., Piffeteau, A., Choquer, M., Malfatti, P., Cimerman, A., Kunz, C., Boccara, M., and Vidal-Cros, A. 2006. Botrytis cinerea virulence is drastically reduced after disruption of chitin synthase class III gene (Bcchs3a). Cell Microbiol. 8:1310-1321.

Sunnarborg, S. W., Miller, S. P., Unnikrishnan, I., and LaPorte, D. C. 2001. Expression of the yeast glycogen phosphorylase gene is regulated by stress-response elements and by the HOG MAP kinase pathway. Yeast 18:1505-1514.

Talbot, N. J. 2003. On the trail of a cereal killer: Exploring the biology of Magnaporthe grisea. Annu. Rev. Microbiol. 57:177-202.

Talbot, N. J., Ebbole, D. J., and Hamer, J. E. 1993. Identification and characterization of $M P G 1$, a gene involved in pathogenicity from the rice blast fungus Magnaporthe grisea. Plant Cell 5:1575-1590.

Tamura, K., Dudley, J., Nei, M., and Kumar, S. 2007. MEGA4: Molecular evolutionary genetics analysis (MEGA) software version 4.0. Mol. Biol. Evol. 24:1596-1599.

Thompson, J. D., Higgins, D. G., and Gibson, T. J. 1994. CLUSTAL W: Improving the sensitivity of progressive multiple sequence alignment through sequence weighting, position-specific gap penalties and weight matrix choice. Nucleic Acids Res. 22:4673-4680

Vendrell, A., Martinez-Pastor, M., Gonzalez-Novo, A., Pascual-Ahuir, A., Sinclair, D. A., Proft, M., and Posas, F. 2011. Sir2 histone deacetylase prevents programmed cell death caused by sustained activation of the Hog1 stress-activated protein kinase. EMBO (Eur. Mol. Biol. Organ.) Rep. 12:1062-1068.

Verna, J., Lodder, A., Lee, K., Vagts, A., and Ballester, R. 1997. A family of genes required for maintenance of cell wall integrity and for the stress response in Saccharomyces cerevisiae. Proc. Natl. Acad. Sci. U.S.A. 94:13804-13809.

Weisman, R., and Choder, M. 2001. The fission yeast TOR homolog, tor $1^{+}$, is required for the response to starvation and other stresses via a conserved serine. J. Biol. Chem. 276:7027-7032.

$\mathrm{Xu}$, J.-R., and Hamer, J. E. 1996. MAP kinase and cAMP signaling regulate infection structure formation and pathogenic growth in the rice blast fungus Magnaporthe grisea. Genes Dev. 10:2696-2706.

Xu, J.-R., Staiger, C. J., and Hamer, J. E. 1998. Inactivation of the mitogen-activated protein kinase Mps1 from the rice blast fungus prevents penetration of host cells but allows activation of plant defense responses. Proc. Natl. Acad. Sci. U.S.A. 95:12713-12718.

Yan, X., Li, Y., Yue, X., Wang, C., Que, Y., Kong, D., Ma, Z., Talbot, N., and Wang, Z. 2011. Two novel transcriptional regulators are essential for infection-related morphogenesis and pathogenicity of the rice blast fungus Magnaporthe oryzae. PLoS Pathog. 7:e1002385. Published online.

Yang, J., Zhang, X., Sun, J., Kang, Z., Ding, S., Xu, J.-R., and Peng, Y. 2010. A novel protein Com1 is required for normal conidium morphology and full virulence in Magnaporthe oryzae. Mol. Plant-Microbe Interact. 23:112-123

Yang, J., Kong, L., Chen, X., Wang, D., Qi, L., Zhao, W., Zhang, Y., Liu, X., and Peng, Y. L. 2012. A carnitine-acylcarnitine carrier protein, MoCrc1, is essential for pathogenicity in Magnaporthe oryzae. Curr. Genet. 58:139-148.

Zhang, H. F, Zhao, Q., Liu, K. Y, Zhang, Z. G, Wang, Y. C, and Zheng, X. B. 2009. $M g C R Z 1$, a transcription factor of Magnaporthe grisea, controls growth, development and is involved in full virulence. FEMS (Fed. Eur. Microbiol. Soc.) Microbiol. Lett. 293:160-169.

Zhang, H. F, Liu, K. Y, Zhang, X., Song, W. W, Zhao, Q., Dong, Y. H, Guo, M., Zheng, X. B, and Zhang, Z. G. 2010a. A two-component histidine kinase, $M o S L N 1$, is required for cell wall integrity and pathogenicity of the rice blast fungus, Magnaporthe oryzae. Curr. Genet. 56:517528.

Zhang, H. F., Liu, K. Y., Zhang, X., Tang, W., Wang, J. S., Guo, M., Zhao, Q., Zheng, X. B., Wang, P., and Zhang, Z. G. 2010b. Two phosphodiesterase genes, $P D E L$ and $P D E H$, regulate development and pathogenicity by modulating intracellular cyclic AMP levels in Magnaporthe oryzae. PLoS One 6:e17241. Published online.

Zhang, H. F, Tang, W., Liu, K. Y, Huang, Q., Zhang, X., Yan, X., Chen, Y., Wang, J. S, Qi, Z. Q, Wang, Z. Y, Zheng, X. B, Wang, P., and Zhang, Z. G. 2011. Eight RGS and RGS-like proteins prchestrate growth, differentiation, and pathogenicity of Magnaporthe oryzae. PLoS Pathog. 7:e1002450. Published online.

Zhou, X. Y., Li, G. T., and Xu, J.-R. 2011a. Efficient approaches for generating GFP fusion and epitope-tagging constructs in filamentous fungi. Pages 199-212 in: Fungal Genomics. J. R. Xu, and H. B. Bluhm, eds. Humana Press, New York.

Zhou, X. Y., Liu, W. D., Wang, C. F., Xu, Q. J., Wang, Y., Ding, S. L., and $\mathrm{Xu}$, J. R. 2011b. A MADS-box transcription factor MoMcm1 is required for male fertility, microconidium production and virulence in Magnaporthe oryzae. Mol. Microbiol. 80:33-53.

Zhou, Z., Li, G., Lin, C., and He, C. 2009. Conidiophore stalk-less1 encodes a putative zinc-finger protein involved in the early stage of conidiation and mycelial infection in Magnaporthe oryzae. Mol. PlantMicrobe Interact. 22:402-410.

\section{AUTHOR-RECOMMENDED INTERNET RESOURCES}

Magnaporthe grisea database:

www.broadinstitute.org/annotation/genome/magnaporthe_grisea/Multi Home.html

GenBank database: www.ncbi.nlm.nih.gov/BLAST

European Molecular Biology Laboratory-European Bioinformatics Institute database: www.ebi.ac.uk 